\title{
Finite volume schemes \\ for locally constrained conservation laws
}

Boris Andreinnov Paola Goatin Nicolas Seguin

March 2, 2009

\begin{abstract}
This paper is devoted to the numerical analysis of the road traffic model proposed by Colombo and Goatin in CG07. The model involves a standard conservation law supplemented by a local unilateral constraint on the flux at the point $x=0$ (modelling a road light, a toll gate, etc.).

We first show that the problem can be interpreted in terms of the theory of conservation laws with discontinuous flux function, as developed by Adimurthi et al. AMG05 and Bürger et al. BKT09. We reformulate accordingly the notion of entropy solution introduced in [CG07, and extend the well-posedness results to the $\mathbf{L}^{\infty}$ framework. Then, starting from a general monotone finite volume scheme for the non-constrained conservation law, we produce a simple scheme for the constrained problem and show its convergence. The proof uses a new notion of entropy process solution. Numerical examples modelling a "green wave" are presented.
\end{abstract}

Key words: Hyperbolic Scalar Conservation Law, Finite Volume Scheme, Entropy Process Solution, Discontinuous Flux, Road Traffic Model

2000 MSC: 35L65 (65M12, 76M12, 90B20)

\section{Contents}

1 Introduction 2

2 Entropy inequalities adapted to the constraint 3

2.1 Definition of solutions and well-posedness in $B V \ldots \ldots$

2.2 Properties and equivalent definitions of solutions $\ldots \ldots \ldots$

$\begin{array}{lll}3 & \text { Entropy process solutions } & 12\end{array}$

$\begin{array}{lll}4 \text { Finite volume schemes } & 15\end{array}$

4.1 Construction of constrained schemes . . . . . . . . . . . . 15

4.2 A priori estimates and discrete entropv inequalities . . . . . . . . 16

4.3 Approximate solutions and convergence . . . . . . . . . . . 18

4.4 A strong compactness approach . . . . . . . . . . . . . . 24

5 Numerical results 24

5.1 A constrained Riemann problem . . . . . . . . . . . . 25

5.2 The green wave . . . . . . . . . . . . . . . . . 26 
6 Appendix: the postponed proofs

\section{Introduction}

The aim of this paper is to study finite volume schemes for scalar conservation laws with local unilateral constraint of the form

$$
\begin{array}{ll}
\partial_{t} u+\partial_{x} f(u)=0, & t>0, x \in \mathbb{R}, \\
u(0, x)=u_{0}(x), & x \in \mathbb{R}, \\
f(u(t, 0)) \leqslant F(t), & t>0 .
\end{array}
$$

We assume that the flux function $f:[0,1] \rightarrow \mathbb{R}$ is Lipschitz continuous with Lipschitz constant $L$ and it is bell-shaped, i.e. it satisfies

$$
f(u) \geqslant 0, \quad f(0)=f(1)=0, \quad f^{\prime}(u)(\bar{u}-u)>0 \quad \text { for a.e. } u \in[0,1],
$$

for some $\bar{u} \in(0,1)$. Accordingly, we assume that $F \in \mathbf{L}^{\infty}\left(\mathbb{R}^{+} ;[0, f(\bar{u})]\right)$ and $u_{0} \in \mathbf{L}^{\infty}(\mathbb{R} ;[0,1])$.

The problem (1.3) was introduced in CG07 to model a toll gate along a highway. It can also model other obstacles on a road, such as road lights (see the numerical examples in Section [5]. The well-posedness of entropy solutions was proved in the $B V$ setting, by means of the wave-front tracking technique.

In the present paper, we characterize and approximate entropy solutions of (113) in the $\mathbf{L}^{\infty}$ setting. The core of the paper is the convergence analysis for finite volume schemes adapted to the constrained problem (13). The schemes are constructed as follows. We consider a classical monotone three-point finite volume scheme (see [EGH00) and denote by $g(u, v)$ the associated numerical flux; at the interface of the mesh which corresponds to the obstacle position $\{x=0\}$, the numerical flux is replaced by $\min (g(u, v), F)$ in order to comply with the constraint (3) (see Section 4 for more details). Our approach is simpler than the wave-front tracking algorithm devised in [CG07, because we do not need to define explicitly the Riemann solvers at the interface $\{x=0\}$ which would fit the constraint at time $t$. Notice that with our approach, existing finite volume codes for the non-constrained conservation law (1) are trivially combined with the constraint (3).

The main difficulty in dealing with the constraint (3) is that the solution may develop stationary non-classical shocks at $x=0$; a non-classical entropy condition is needed in order to govern the admissibility of weak solutions. Moreover, in our context the corresponding entropy condition must be combined with the usual numerical analysis techniques for the scalar conservation law.

The first definition of entropy solutions was introduced in CG07 (it was derived from the entropy condition associated to conservation laws with discontinuous flux function, see e.g. Tow00]). It turns out that with the definition of [CG07, proving the stability of entropy solutions is delicate. More precisely, even under strong compactness assumptions on a sequence $\left(u^{\varepsilon}\right)_{\varepsilon>0}$ of entropy solutions to (11), (3), it is not straightforward that the limit $u$ of $u^{\varepsilon}$ is also an entropy solution to (11), (31). Indeed, the constraint (3) on the flux traces is not contained in the entropy inequalities of [CG07, but it has to be checked apart. 
The passage to the limit in this constraint is only possible in an indirect way, e.g. using the Green-Gauss formula (see (7) below). The situation is far more delicate if only weak compactness of $\left(u^{\varepsilon}\right)_{\varepsilon>0}$ is available.

Therefore, although the theory of [CG07] leads to well-posedness of (13), for the purpose of proving convergence of the aforementioned numerical schemes we prefer to give a new "global" definition of $\mathcal{G}$-entropy solutions. Then we define the corresponding $\mathcal{G}$-entropy process solutions in the spirit of [EGH00] (this generalization is needed whenever one lacks strong compactness for the sequence of approximate solutions). In particular, the global entropy inequalities have the advantage of implying the constraint (3). The notion of $\mathcal{G}$-entropy solution is clearly stable under the strong $\mathbf{L}_{\text {loc }}^{1}$ convergence, and an $\mathbf{L}^{\infty}$ weak- $\star$ limit of a sequence of $\mathcal{G}$-entropy solutions is a $\mathcal{G}$-entropy process solution.

It is worth noting that, unlike the standard cases EGH00, we are not able to prove the uniqueness of $\mathscr{G}$-entropy process solutions directly. Therefore, in order to prove the convergence of the scheme we need to know beforehand the existence of a $\mathscr{G}$-entropy solution (guaranteed by the results of CG07).

We study the constrained conservation law (10), (3) with the tools developed in the context of conservation laws with discontinuous flux. Our definitions are based upon the notion of " $(A, B)$-connection" (see Adimurthi, Mishra and Veerappa Gowda AMG05] and Bürger, Karlsen and Towers [BKT09]) and upon the idea of "adapted entropies" (see Baiti and Jenssen [BJ97] and Audusse and Perthame AuP05) further developed in the forthcoming work of Andreianov, Karlsen and Risebro AKR.

The paper is organized as follows. In Section 2 we define $\mathcal{G}$-entropy solutions, show that they coincide with the entropy solutions in the sense of Colombo and Goatin [CG07, and extend the well-posedness results of [CG07 to the case of $\mathbf{L}^{\infty}$ data. Some comments on the notion of $\mathcal{G}$-entropy solution are given in Remarks 45] A key lemma and the technical proofs are postponed to the Appendix. Then in Section 3 we describe the entropy-process solutions. Section 4 is devoted to the construction of finite volume schemes for (10 3) and to the proof of their convergence; at the end of the section, the main result of the paper (Theorem 4.9) is stated. Section 5 contains numerical examples.

\section{Entropy inequalities adapted to the constraint}

In this section, we study the entropy solutions for the Cauchy problem (13). We give several notions of solution; the fact that all these notions are equivalent is the main point of the section. Starting from the $B V$ existence result of [CG07, we then deduce well-posedness (existence, uniqueness, $\mathbf{L}^{1}$ stability with respect to the perturbation of $u_{0}$ and of $F$ ) for (13) in the framework of the entropy solutions. Notice that the uniqueness and the existence proofs below exploit different definitions of entropy solution.

\subsection{Definition of solutions and well-posedness in $B V$}

Let us note $\Pi=[0,+\infty) \times \mathbb{R}$. Throughout the paper,

$$
\Phi:(u, \kappa) \in \mathbb{R} \times \mathbb{R} \mapsto \operatorname{sgn}(u-\kappa)(f(u)-f(\kappa))
$$


denotes the entropy flux associated with the Kruzhkov entropy $(u, \kappa) \mapsto|u-\kappa|$ (see Kru70]). Following CG07, we give the following definition of entropy solutions of (1.3).

Definition 2.1. Let $u_{0} \in \mathbf{L}^{\infty}(\mathbb{R} ;[0,1])$ and $F \in \mathbf{L}^{\infty}\left(\mathbb{R}^{+} ;[0, f(\bar{u})]\right)$. A function $u \in \mathbf{L}^{\infty}(\Pi ;[0,1])$ is said to be a CG-entropy solution of (13) if

(i) for all nonnegative test function $\varphi \in \mathcal{C}_{c}^{\infty}(\Pi)$ and all $\kappa \in[0,1]$,

$$
\begin{aligned}
& \int_{0}^{+\infty} \int_{\mathbb{R}}\left(|u(t, x)-\kappa| \partial_{t}+\Phi(u(t, x), \kappa) \partial_{x}\right) \varphi(t, x) d x d t \\
+ & \int_{\mathbb{R}}\left|u_{0}(x)-\kappa\right| \varphi(0, x) d x+2 \int_{0}^{+\infty}(1-F(t) / f(\bar{u})) f(\kappa) \varphi(t, 0) d t \geqslant 0
\end{aligned}
$$

(ii) the constraint (3) on the traces of $f(u(t, \cdot))$ on $\{x=0\}$ is satisfied:

$$
f\left(\left(\gamma^{l} u\right)(t)\right)=f\left(\left(\gamma^{r} u\right)(t)\right) \leqslant F(t) \quad \text { for a.e. } t>0,
$$

where $\gamma^{l, r}$ denote the operators of left- and right-side strong traces on $\{x=0\}$.

Here and in the sequel, we use e.g. $\mathbf{L}^{\infty}(\Pi,[0,1])$ for the set of all measurable functions with values in $[0,1]$, etc..

Remark 1. Taking $\kappa=0$, then $\kappa=1$ in the above formulation (15), from the condition $u(t, x) \in[0,1]$ a.e. we deduce that $u$ is a weak solution of equation (11) (i.e., $u$ is a solution in the sense of distributions). In particular, the equality contained in (6) is the classical Rankine-Hugoniot condition which can be derived from the weak formulation of the conservation law (11).

Remark 2. The inequality contained in the constraint (6) can be rewritten in the following weak form:

$$
\begin{aligned}
& \int_{0}^{+\infty} F(t) \xi(t) d t \geqslant \int_{0}^{+\infty} f\left(\left(\gamma^{l, r} u\right)(t)\right) \xi(t) d t \\
&= \pm \int_{0}^{+\infty} \int_{\mathbb{R}^{ \pm}}\left(u(t, x) \partial_{t}+f(u(t, x)) \partial_{x}\right)(\xi(t) \varphi(x)) d x d t
\end{aligned}
$$

for all $\xi \in \mathcal{C}_{c}^{\infty}((0,+\infty)), \xi \geqslant 0$, and some given $\varphi \in \mathcal{C}_{c}^{\infty}(\mathbb{R}), \varphi \geqslant 0, \varphi(0)=1$.

It is worth noting that the traces $\gamma^{l, r} u$ in (6) do exist. Indeed, we are dealing with a flux function such that the measure of the set $\left\{s \in[0,1], f^{\prime}(s)=0\right\}$ is zero (by assumption (4)). In this situation, the following result was shown by Panov (see also Vasseur Vas01).

Theorem 2.2 (Adapted from Pan07). Let $f$ be a continuous function which is non constant on any non-degenerate interval of $[0,1]$. Let $u$ be an entropy solution of $\partial_{t} u+\partial_{x} f(u)=0$ in $(0,+\infty) \times(0,+\infty)$. Then there exists a strong trace $\gamma^{r} u$ on the boundary $\{x=0\}$, in the sense

$$
\lim _{h \downarrow 0} \frac{1}{h} \int_{0}^{+\infty} \int_{0}^{h}\left|u(t, x)-\left(\gamma^{r} u\right)(t)\right| \xi(t) d x d t=0 \quad \forall \xi \in \mathcal{C}_{c}^{\infty}([0,+\infty)) .
$$


The existence of the left-sided trace $\gamma^{l} u$ of an entropy solution $u$ of $\partial_{t} u+$ $\partial_{x} f(u)=0$ in $(0,+\infty) \times(-\infty, 0)$ is analogous (it can also be deduced from the above statement, upon changing $x, f$ into $-x,-f$, respectively).

Note that for all continuous functions $\theta$ on $[0,1]$, equality (8) implies

$$
\lim _{h \downarrow 0} \frac{1}{h} \int_{0}^{+\infty} \int_{0}^{h}\left|\theta \circ u(t, x)-\left(\theta \circ \gamma^{r} u\right)(t)\right| \xi(t) d x d t=0 \quad \forall \xi \in \mathcal{C}_{c}^{\infty}([0,+\infty)),
$$

i.e., $\theta \circ\left(\gamma^{r} u\right)$ is the strong trace $\gamma^{r}(\theta \circ u)$ of $\theta \circ u$. For the proof, it is sufficient to take a concave modulus of continuity $M_{\theta}(\cdot)$ of $\theta$ and use the Jensen inequality:

$$
\begin{aligned}
& \frac{1}{h} \int_{0}^{+\infty} \int_{0}^{h}\left|(\theta \circ u)(t, x)-\left(\theta \circ \gamma^{r} u\right)(t)\right| \xi(t) d x d t \\
& \leqslant \frac{1}{h} \int_{0}^{+\infty} \int_{0}^{h} M_{\theta}\left(\left|u(t, x)-\left(\gamma^{r} u\right)(t)\right|\right) \xi(t) d x d t \\
\leqslant & \left(\int_{0}^{+\infty} \xi(t) d t\right) \times M_{\theta}\left(\frac{1}{\int_{0}^{+\infty} \xi(t) d t} \frac{1}{h} \int_{0}^{+\infty} \int_{0}^{h}\left|(\theta \circ u(t, x))-\left(\theta \circ \gamma^{r} u\right)(t)\right| \xi(t) d x d t\right),
\end{aligned}
$$

so that (9) is implied by (8) and the fact that $M_{\theta}(r) \rightarrow 0$ as $r \rightarrow 0$.

Remark 3. The last term of (5) can be seen as the remainder of the approximation of Problem (1.3) by

$$
\begin{aligned}
& \partial_{t} u^{\varepsilon}(t, x)+\partial_{x}\left(k^{\varepsilon}(t, x) f\left(u^{\varepsilon}\right)\right)=0, \quad t>0, x \in \mathbb{R} \\
& \text { where } k^{\varepsilon}(t, x)= \begin{cases}1 & \text { if }|x| \geqslant \varepsilon, \\
F(t) / f(\bar{u}) & \text { if }|x|<\varepsilon .\end{cases}
\end{aligned}
$$

The associated entropy formulation (see for instance Tow00, KRT03, SV03, BV06]) is

$$
\begin{aligned}
\int_{0}^{+\infty} \int_{\mathbb{R}}\left(\left|u^{\varepsilon}(t, x)-\kappa\right|\right. & \left.\partial_{t}+\Phi\left(u^{\varepsilon}(t, x), \kappa\right) \partial_{x}\right) \varphi(t, x) d x d t \\
& +\int_{\mathbb{R}}\left|u_{0}(x)-\kappa\right| \varphi(0, x) d x \\
+ & \int_{0}^{+\infty}(1-F(t) / f(\bar{u})) f(\kappa)(\varphi(t,-\varepsilon)+\varphi(t, \varepsilon)) d t \geqslant 0
\end{aligned}
$$

clearly, the solution $u^{\varepsilon}$ satisfies the constraint $f\left(u^{\varepsilon}(t, x)\right) \leqslant F(t)$ for a.e. $|x|<\varepsilon$.

Problem (13) is well-posed in the framework of CG-entropy solutions of bounded variation. More precisely, we recall the following result.

Theorem 2.3 (Colombo and Goatin, CG07).

Assume that $u_{0} \in B V(\mathbb{R},[0,1])$ and $F \in B V\left(\mathbb{R}^{+},[0, f(\bar{u})]\right)$. Then there exists one and only one $C G$-entropy solution $u \in B V(\Pi)$ to Problem (13). Moreover, given two initial data $u_{0}, v_{0} \in B V(\mathbb{R},[0,1])$ such that $\left(u_{0}-v_{0}\right) \in \mathbf{L}^{1}(\mathbb{R})$, the corresponding $C G$-entropy solutions $u, v$ satisfy the following $\mathbf{L}^{1}$-stability property:

$$
\int_{\mathbb{R}}|u(t, x)-v(t, x)| d x \leqslant \int_{\mathbb{R}}\left|u_{0}(x)-v_{0}(x)\right| d x .
$$


In [CG07, existence is proved using the wave-front tracking approximation and a Temple functional. Note that, in the case of wave-front tracking approximations (as well as in the case of the approximations of Remark 3), the traces $\gamma^{l, r} f(u)$ of the limit of $f\left(u^{\varepsilon}\right)$ are the limits of the traces $\gamma^{l, r} f\left(u^{\varepsilon}\right)$, respectively, thanks to the Green-Gauss formula (cf. (7)). Hence (6) in Definition 2.1(ii) is verified. Uniqueness is obtained using the Kruzhkov doubling of variables technique ( Kru70] ) on each side from the boundary; the constraint (6) for the strong traces of $u$ is used to obtain the dissipativity inequalities across the interface $\{x=0\}$.

In the next Section, this existence and uniqueness result will be extended to $\mathbf{L}^{\infty}$ data (as well for $F$ as for $u_{0}$ ) and solutions; see Theorem 2.11

\subsection{Properties and equivalent definitions of solutions}

Recall that, according to Theorem 2.2 for each function $u \in \mathbf{L}^{\infty}(\Pi)$ such that $u$ is a Kruzhkov entropy solution away from the interface $\{x=0\}$ (i.e., such that (5) holds with test functions $\left.\varphi \in \mathcal{C}_{c}^{\infty}(\Pi),\left.\varphi\right|_{\{x=0\}} \equiv 0\right)$, one can define a couple of strong one-sided traces $\left(\left(\gamma^{l} u\right)(t),\left(\gamma^{r} u\right)(t)\right)$, for a.e. $t>0$. The starting point of the below constructions is the explicit description of the set of possible values taken by such couples of one-sided traces. Define the following sets.

Definition 2.4. Let $F \in[0, f(\bar{u})]$. The admissibility germ $\mathcal{G}(F)$ for the conservation law (11) associated with the constraint $\left.f(u)\right|_{\{x=0\}} \leqslant F$ is the subset of $[0,1]^{2}$ defined as the union $\mathscr{G}(F)=\mathscr{G}_{1}(F) \cup \mathscr{G}_{2}(F) \cup \mathscr{G}_{3}(F)$, where

- $\mathscr{G}_{1}(F)=\left\{\left(c_{l}, c_{r}\right) \in[0,1]^{2} ; c_{l}>c_{r}, f\left(c_{l}\right)=f\left(c_{r}\right)=F\right\}$,

- $\mathscr{G}_{2}(F)=\left\{(c, c) \in[0,1]^{2} ; f(c) \leqslant F\right\}$,

- $\mathscr{G}_{3}(F)=\left\{\left(c_{l}, c_{r}\right) \in[0,1]^{2} ; c_{l}<c_{r}, f\left(c_{l}\right)=f\left(c_{r}\right) \leqslant F\right\}$,

Remark that $\mathscr{G}_{1}(F)=\left\{\left(A_{F}, B_{F}\right)\right\}$ is a singleton, uniquely defined by the conditions

$$
f\left(A_{F}\right)=f\left(B_{F}\right)=F, \quad A_{F} \geqslant B_{F} .
$$

The fundamental property of CG-entropy solutions is the following.

Proposition 2.5. If $u$ is a CG-entropy solution or Problem (1] 3), then, for a.e. $t>0$,

$$
\left(\left(\gamma^{l} u\right)(t),\left(\gamma^{r} u\right)(t)\right) \in \mathscr{G}(F(t))
$$

Proof. First, recall that Definition 2.1 contains the fact that the traces $\gamma^{l, r} u$ satisfy the Rankine-Hugoniot condition at the interface $\{x=0\}$ : for a.e. $t>0$,

$$
f\left(\left(\gamma^{l} u\right)(t)\right)=f\left(\left(\gamma^{r} u\right)(t)\right)
$$

In order to prove (10), it suffices to show that the non classical shock

$$
f\left(\left(\gamma^{l} u\right)(t)\right)=f\left(\left(\gamma^{r} u\right)(t)\right)<F(t) \text { with }\left(\gamma^{l} u\right)(t)>\left(\gamma^{r} u\right)(t)
$$

is excluded, for a.e. $t>0$. Indeed, notice that the Rankine-Hugoniot condition (11) and the assumptions (4) on the flux function $f$ yield, for a.e. $t>0$, the following alternative:

$$
\text { either }\left(\left(\gamma^{l} u\right)(t),\left(\gamma^{r} u\right)(t)\right) \in \mathscr{G}(F(t)) \text {, or (12) holds. }
$$


To contradict (12), consider a nonnegative test function $\xi \in \mathcal{C}_{c}^{\infty}((0,+\infty))$ and take $\varphi_{\varepsilon}=w_{\varepsilon} \xi$ with $\varepsilon>0$ in (5). Here $w_{\varepsilon}$ is the cut-off function defined by

$$
w_{\varepsilon}(x)= \begin{cases}1 & \text { if }|x|<\varepsilon, \\ 2-|x| / \varepsilon & \text { if } \varepsilon \leqslant|x| \leqslant 2 \varepsilon, \\ 0 & \text { if }|x|>2 \varepsilon .\end{cases}
$$

Then the entropy inequality (15) becomes

$$
\begin{gathered}
I(\varepsilon)+J(\varepsilon) \geqslant 0, \\
I(\varepsilon)=\int_{0}^{+\infty} \int_{\mathbb{R}}\left(|u-\kappa| \partial_{t} \xi+\Phi(u, \kappa) \partial_{x} \xi\right) w_{\varepsilon} d x d t, \\
J(\varepsilon)=\int_{0}^{+\infty} \int_{\mathbb{R}} \Phi(u, \kappa) \xi w_{\varepsilon}^{\prime} d x d t x+2 \int_{0}^{+\infty}(1-F(t) / f(\bar{u})) f(\kappa) \xi(t) d t .
\end{gathered}
$$

Clearly, $\lim _{\varepsilon \rightarrow 0} I(\varepsilon)=0$. Moreover, using (9) with $\theta(\cdot)=\Phi(\cdot, \kappa)$, we deduce

$$
\begin{aligned}
\lim _{\varepsilon \rightarrow 0} J(\varepsilon)=\int_{0}^{+\infty}\left(\Phi\left(\left(\gamma^{l} u\right)(t), \kappa\right)-\Phi\left(\left(\gamma^{r} u\right)(t), \kappa\right)\right. & \\
& +2(1-F(t) / f(\bar{u})) f(\kappa)) \xi(t) d t
\end{aligned}
$$

which gives for all $\kappa \in[0,1]$ and a.e. $t>0$

$$
\Phi\left(\left(\gamma^{l} u\right)(t), \kappa\right)-\Phi\left(\left(\gamma^{r} u\right)(t), \kappa\right)+2(1-F(t) / f(\bar{u})) f(\kappa) \geqslant 0 .
$$

Let us take $\kappa=\bar{u}$ in the latter expression. Assuming (12), we obtain

$$
\begin{aligned}
0 & \leqslant \Phi\left(\left(\gamma^{l} u\right)(t), \bar{u}\right)-\Phi\left(\left(\gamma^{r} u\right)(t), \bar{u}\right)+2(1-F(t) / f(\bar{u})) f(\bar{u}) \\
& \leqslant f\left(\left(\gamma^{l} u\right)(t)\right)-f(\bar{u})-f(\bar{u})+f\left(\left(\gamma^{r} u\right)(t)\right)+2(f(\bar{u})-F(t)), \\
& \leqslant 2\left(f\left(\left(\gamma^{l} u\right)(t)\right)-F(t)\right),
\end{aligned}
$$

which is in contradiction with (12).

Using the ideas of Baiti and Jenssen BJ97, Audusse and Perthame AuP05 and Bürger, Karlsen and Towers [BKT09] (see also the forthcoming work of Andreianov, Karlsen and Risebro AKR and Remarks 45 below), we can propose several other ways to define entropy solutions for the constrained problem (113). One definition exploits the explicit description (10) of the possible one-sided traces of $u$ at $\{x=0\}$. The other one is based upon the comparison of the solution with functions $c$ defined by

$$
c(x):=c_{l} \mathbb{1}_{\{x<0\}}+c_{r} \mathbb{1}_{\{x>0\}} \equiv \begin{cases}c_{l} & \text { if } x<0, \\ c_{r} & \text { if } x>0,\end{cases}
$$

with $\left(c_{l}, c_{r}\right) \in[0,1]^{2}$ (here, the couples $\left(c_{l}, c_{r}\right) \in \mathcal{G}(F(t)$ ), which lead to admissible stationary weak solutions (14) to (10, (3), play a central role). We gather the definitions and the fact that they are equivalent into one proposition.

Proposition 2.6. Let $u_{0} \in \mathbf{L}^{\infty}(\mathbb{R},[0,1])$ and $F \in \mathbf{L}^{\infty}\left(\mathbb{R}^{+},[0, f(\bar{u})]\right)$. Let $u \in$ $\mathbf{L}^{\infty}(\Pi ;[0,1])$. Then the assertions $(\mathbf{A}),(\mathbf{B})$ and $(\mathbf{C})$ below are equivalent: 
(A) $\left(A_{1}\right) u$ is a Kruzhkov entropy solution for $x<0$ and $x>0$, i.e., for all nonnegative test functions $\varphi \in \mathcal{C}_{c}^{\infty}(\Pi \backslash\{x=0\})$ and all $\kappa \in[0,1]$,

$$
\begin{aligned}
\int_{0}^{+\infty} \int_{\mathbb{R}}\left(|u(t, x)-\kappa| \partial_{t}+\right. & \left.\Phi(u(t, x), \kappa) \partial_{x}\right) \varphi(t, x) d x d t \\
& +\int_{\mathbb{R}}\left|u_{0}(x)-\kappa\right| \varphi(0, x) d x \geqslant 0
\end{aligned}
$$

$\left(A_{2}\right)$ in addition, for a.e. $t>0$,

$$
\left(\left(\gamma^{l} u\right)(t),\left(\gamma^{r} u\right)(t)\right) \in \mathscr{G}(F(t)) .
$$

(B) $\left(B_{1}\right)$ assertion $\left(A_{1}\right)$ holds, i.e., $u$ is a Kruzhkov entropy solution for $x<0$ and $x>0$, in the sense (15);

$\left(B_{2}\right) u$ is a weak solution of (1), i.e., for all test functions $\varphi \in \mathcal{C}_{c}^{\infty}(\Pi)$, $\varphi(0, x)=0$,

$$
\int_{0}^{+\infty} \int_{\mathbb{R}}\left(u(t, x) \partial_{t}+f(u(t, x)) \partial_{x}\right) \varphi(t, x) d x d t=0 ;
$$

$\left(B_{3}\right)$ in addition, for a.e. $t>0$,

$$
\forall\left(c_{l}, c_{r}\right) \in \mathscr{G}(F(t)) \quad \Phi\left(\left(\gamma^{l} u\right)(t), c_{l}\right) \geqslant \Phi\left(\left(\gamma^{r} u\right)(t), c_{r}\right) .
$$

(C) u satisfies the following "global" entropy inequalities:

$\left(C_{1}\right)$ there exists $M>0$ such that for all $\left(c_{l}, c_{r}\right) \in[0,1]^{2}$ and all nonnegative test functions $\varphi \in \mathcal{C}_{c}^{\infty}(\Pi)$,

$$
\begin{aligned}
\int_{0}^{+\infty} \int_{\mathbb{R}}(\mid u(t, x)- & \left.c(x) \mid \partial_{t}+\Phi(u(t, x), c(x)) \partial_{x}\right) \varphi(t, x) d x d t \\
& +\int_{\mathbb{R}}\left|u_{0}(x)-c(x)\right| \varphi(0, x) d x \\
\geqslant & -M \int_{0}^{+\infty} \operatorname{dist}\left(\left(c_{l}, c_{r}\right), \mathcal{G}(F(t))\right) \varphi(t, 0) d t
\end{aligned}
$$

where $c(x)$ is the piecewise constant function given by (14), and dist refers to a distance function on $\mathbb{R}^{2}$.

Notice that the right-hand side of (19) makes sense. Indeed, one easily shows that the map $F \mapsto\left(A_{F}, B_{F}\right)$ is continuous, and the continuity of the map $F \mapsto \operatorname{dist}\left(\left(c_{l}, c_{r}\right), \mathcal{G}(F)\right)$ follows; in addition, the map $t \mapsto F(t)$ is measurable by assumption. Thus $t \mapsto \operatorname{dist}\left(\left(c_{l}, c_{r}\right), \mathcal{G}(F(t))\right)$ is an $\mathbf{L}^{\infty}((0,+\infty))$ function.

The proof of the equivalence of (A), (B) and (C) relies on the "dissipativity" and the "maximality" properties of the admissibility germ $\mathcal{G}(F)$ stated in the following lemma.

Lemma 2.7.

(i) If $\left(b_{l}, b_{r}\right) \in \mathscr{G}(F)$, then

$$
\forall\left(c_{l}, c_{r}\right) \in \mathscr{G}(F), \quad \Phi\left(b_{l}, c_{l}\right) \geqslant \Phi\left(b_{r}, c_{r}\right) .
$$


(ii) The converse is true, under the following form:

$$
\begin{aligned}
& \text { if (20) holds and the Rankine-Hugoniot condition } \\
& f\left(b_{l}\right)=f\left(b_{r}\right) \text { is satisfied, then }\left(b_{l}, b_{r}\right) \in \mathscr{G}(F) \text {. }
\end{aligned}
$$

Lemma 2.7 expresses the essential structural properties of the set $\mathscr{G}(F)$. In the two remarks that follow, we comment the result of the lemma and explain the particular role played by $\mathscr{G}_{1}(F)$.

Remark 4. Denote by $\mathcal{H}$ any of the sets $\mathscr{G}_{1}(F), \mathscr{G}_{1}(F) \cup \mathscr{G}_{2}(F)$, or $\mathscr{G}(F) \equiv$ $\mathscr{G}_{1}(F) \cup \mathscr{G}_{2}(F) \cup \mathscr{G}_{3}(F)$. Then $\mathcal{H}$ possesses the following three properties:

(a) the Rankine-Hugoniot condition is satisfied:

$$
\forall\left(c_{l}, c_{r}\right) \in \mathcal{H}, \quad f\left(c_{l}\right)=f\left(c_{r}\right) ;
$$

(b) the dissipativity condition is fulfilled:

$$
\forall\left(c_{l}, c_{r}\right),\left(b_{l}, b_{r}\right) \in \mathcal{H}, \quad \Phi\left(c_{l}, b_{l}\right) \geqslant \Phi\left(c_{r}, b_{r}\right) ;
$$

(c) among all extensions of $\mathcal{H}$ that still possess the two aforementioned properties, there exists the greatest extension $\mathcal{H}^{*}$ determined by

$$
\mathcal{H}^{*}:=\left\{\left(b_{l}, b_{r}\right) \text { such that } f\left(b_{l}\right)=f\left(b_{r}\right) \mid \forall\left(c_{l}, c_{r}\right) \in \mathcal{H}, \Phi\left(c_{l}, b_{l}\right) \geqslant \Phi\left(c_{r}, b_{r}\right)\right\} .
$$

It should be understood that the fact that $\mathcal{H}^{*}$ satisfies the "dissipativity property" (b) is equivalent to the existence of a unique maximal extension of $\mathcal{H}$, as stated in (c). In each of the three cases under consideration, we have $\mathcal{H}^{*}=\mathcal{G}(F)$.

Remark 5 . In the case $F(t) \equiv$ const, it can be easily seen that $(\mathbf{C})$ is equivalent to

$$
\left\{\begin{array}{l}
\text { (19) with } \varphi \in \mathcal{C}_{c}^{\infty}\left(\mathbb{R}^{+} \times \mathbb{R}^{*}\right) \text { and any }\left(c_{l}, c_{r}\right) \in[0,1]^{2}, \\
\text { (19) with } \varphi \in \mathcal{C}_{c}^{\infty}\left(\mathbb{R}^{+} \times \mathbb{R}\right) \text { and }\left(c_{l}, c_{r}\right) \in \mathscr{G}(F) \text {. }
\end{array}\right.
$$

Let us stress that in both cases in (22), the remainder term of (19) vanishes. Furthermore, according to Remark 4 it is sufficient to replace $\mathscr{G}(F)$ in (22) by $\mathscr{G}_{1}(F)$ (i.e., to use (19) with only one couple $\left(c_{l}, c_{r}\right)=(A(F), B(F))$, when $\varphi$ does not vanish on $\{x=0\})$. The so obtained definition of a $\mathcal{G}$-entropy solution reduces to the definition of Bürger, Karlsen and Towers BKT09; it corresponds to the choice of the $(A, B)$-connection $(A(F), B(F))$.

We postpone to Appendix the proofs of Lemma 2.7 and of Proposition 2.6

Definition 2.8. If any of the properties (A), (B) or (C) of Proposition 2.6 holds, then $u$ is called a $\mathcal{G}$-entropy solution of Problem (13).

Actually, Definition 2.1 and Definition 2.8 are equivalent:

Theorem 2.9. A function $u \in \mathbf{L}^{\infty}(\Pi)$ is a $\mathscr{G}$-entropy solution if and only if it is a $C G$-entropy solution.

Proof. Let us first prove that a CG-entropy solution is a $\mathscr{G}$-entropy solution; we verify property (A) of Proposition 2.6 Indeed, $\left(A_{1}\right)$ is a direct consequence of Definition 2.11(i); it suffices to take $\varphi \in \mathcal{C}_{c}^{\infty}(\Pi \backslash\{x=0\})$ in (5). The second point $\left(A_{2}\right)$ has already been stated in Proposition 2.5 
We now justify the converse statement. Assume that $u$ is a $\mathscr{G}$-entropy solution (once more, we use the formulation $(\mathbf{A})$ ). In view of $\left(A_{2}\right)$ and Definition 2.4 of $\mathcal{G}(F(t))$, the constraint (6) of Definition 2.1(ii) is satisfied. It remains to justify (5) in Definition 2.1(i). Consider a nonnegative test function $\varphi \in \mathcal{C}_{c}^{\infty}\left(\mathbb{R}^{+} \times \mathbb{R}\right)$ and denote $\varphi_{\varepsilon}^{1}=\left(1-w_{\varepsilon}\right) \varphi$ and $\varphi_{\varepsilon}^{2}=w_{\varepsilon} \varphi$ with $w_{\varepsilon}$ defined by (13), then $\varphi=\varphi_{\varepsilon}^{1}+\varphi_{\varepsilon}^{2}$. Therefore,

$$
\begin{aligned}
I:= & \int_{\mathbb{R}^{+}} \int_{\mathbb{R}}\left(|u-\kappa| \partial_{t}+\Phi(u, \kappa) \partial_{x}\right) \varphi d x d t \\
& +\int_{\mathbb{R}}\left|u_{0}(x)-\kappa\right| \varphi(0, x) d x+2 \int_{\mathbb{R}^{+}}(1-F(t) / f(\bar{u})) f(\kappa) \varphi(t, 0) d t \\
= & \int_{\mathbb{R}^{+}} \int_{\mathbb{R}}\left(|u-\kappa| \partial_{t}+\Phi(u, \kappa) \partial_{x}\right) \varphi_{\varepsilon}^{1} d x d t+\int_{\mathbb{R}}\left|u_{0}(x)-\kappa\right| \varphi_{\varepsilon}^{1}(0, x) d x \\
& +\int_{\mathbb{R}^{+}} \int_{\mathbb{R}} w_{\varepsilon}\left(|u-\kappa| \partial_{t}+\Phi(u, \kappa) \partial_{x}\right) \varphi d x d t \\
& -\frac{1}{\varepsilon} \int_{\mathbb{R}^{+}} \int_{\varepsilon<|x|<2 \varepsilon} \Phi(u(t, x), \kappa) \operatorname{sgn}(x) \varphi d x d t \\
& +\int_{\mathbb{R}^{\prime}}\left|u_{0}(x)-\kappa\right| \varphi_{\varepsilon}^{2}(0, x) d x+2 \int_{\mathbb{R}^{+}}(1-F(t) / f(\bar{u})) f(\kappa) \varphi(t, 0) d t .
\end{aligned}
$$

If $u$ is a $\mathscr{G}$-entropy solution, then the sum of the two first terms of the right-hand side above is nonnegative by (15), since $\varphi_{\varepsilon}^{1} \in \mathcal{C}_{c}^{\infty}(\Pi \backslash\{x=0\})$. Now, letting $\varepsilon$ tend to 0 and using the strong trace property (9), we obtain

$$
I \geqslant \int_{\mathbb{R}^{+}} B(t, \kappa) \varphi(t, 0) d t
$$

where

$$
B(t, \kappa) \geqslant \Phi\left(\left(\gamma^{l} u\right)(t), \kappa\right)-\Phi\left(\left(\gamma^{r} u\right)(t), \kappa\right)+2(1-F(t) / f(\bar{u})) f(\kappa) .
$$

In order to conclude the proof, let us show that $B(t, \kappa)$ is non-negative for a.e. $t>0$. The proof is a case by case study. Let us fix $t_{0}>0$ and consider $\left(\left(\gamma^{l} u\right)\left(t_{0}\right),\left(\gamma^{r} u\right)\left(t_{0}\right)\right) \in \mathcal{G}\left(F\left(t_{0}\right)\right)$ (whenever this is convenient, we drop the dependence in $t_{0}$ in the subsequent notation).

If $\left(\gamma^{l} u, \gamma^{r} u\right) \in \mathscr{G}_{2}(F) \cup \mathscr{G}_{3}(F)$, then the standing wave

$$
\tilde{u}(t, x):=\left(\gamma^{l} u\right)\left(t_{0}\right) \mathbb{1}_{\{x<0\}}+\left(\gamma^{r} u\right)\left(t_{0}\right) \mathbb{1}_{\{x>0\}}
$$

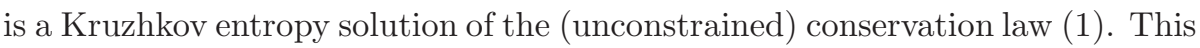
amounts to say that for all $\kappa \in[0,1]$,

$$
\Phi\left(\left(\gamma^{l} u\right), \kappa\right)-\Phi\left(\left(\gamma^{r} u\right), \kappa\right) \geqslant 0 .
$$

Since the last term in (23) is non-negative, we have $B\left(t_{0}, \kappa\right) \geqslant 0$. Now, we consider the case $\left(\gamma^{l} u, \gamma^{r} u\right) \in \mathscr{G}_{1}(F)$, i.e., $\gamma^{l} u \geqslant \gamma^{r} u$ and $f\left(\gamma^{l} u\right)=f\left(\gamma^{r} u\right)=F$.

- If $0 \leqslant \kappa \leqslant \gamma^{r} u$, then

$$
\Phi\left(\gamma^{l} u, \kappa\right)-\Phi\left(\gamma^{r} u, \kappa\right)=f\left(\gamma^{l} u\right)-f(\kappa)-f\left(\gamma^{r} u\right)+f(\kappa)=0 .
$$


- If $\gamma^{r} u \leqslant \kappa \leqslant \gamma^{l} u$, then

$$
\begin{aligned}
\Phi\left(\gamma^{l} u, \kappa\right) & -\Phi\left(\gamma^{r} u, \kappa\right)+2(1-F / f(\bar{u})) f(\kappa) \\
& =f\left(\gamma^{l} u\right)-f(\kappa)+f\left(\gamma^{r} u\right)-f(\kappa)+2 f(\kappa)-2 F f(\kappa) / f(\bar{u}) \\
& =2 F(1-f(\kappa) / f(\bar{u})) \geqslant 0 .
\end{aligned}
$$

- If $\gamma^{l} u \leqslant \kappa \leqslant 1$, then

$$
\Phi\left(\gamma^{l} u, \kappa\right)-\Phi\left(\gamma^{r} u, \kappa\right)=f(\kappa)-f\left(\gamma^{l} u\right)-f(\kappa)+f\left(\gamma^{r} u\right)=0 .
$$

Hence, $B\left(t_{0}, \kappa\right)$ is non-negative in all cases. Thus $I \geqslant 0$, which ends the proof.

Now, recall the following $\mathbf{L}^{1}$ continuous dependence and finite speed of propagation property (adapted from Kru70). Let $T>0, m>L T$ (where $L$ is the Lipschitz constant of $f$ ), $M>m$. Let $u, v$ be entropy solutions of (11)(2) in the sense of [15]), corresponding to the initial data $u_{0}, v_{0} \in \mathbf{L}^{\infty}(\mathbb{R} ;[0,1])$, respectively. Then for a.e. $T>0$, we have

$$
\int_{\{m<|x|<M\}}|u-v|(T, x) d x \leqslant \int_{\{m-L T<|x|<M+L T\}}\left|u_{0}-v_{0}\right|(x) d x .
$$

We complement this property with the following one, which takes into account the constraint at $\{x=0\}$.

Proposition 2.10. Assume that $F^{1}, F^{2} \in \mathbf{L}^{\infty}\left(\mathbb{R}^{+} ;[0, f(\bar{u})]\right)$, and $u_{0}, v_{0} \in$ $\mathbf{L}^{\infty}(\mathbb{R},[0,1])$ such that $\left(u_{0}-v_{0}\right) \in \mathbf{L}^{1}(\mathbb{R})$. Assume that $u$, $v$ are $C G$-entropy solutions of Problem (113) corresponding to the initial data $u_{0}, v_{0}$ and to the constraints $F^{1}, F^{2}$, respectively. Then, for a.e. $T>0$, we have

$$
\int_{\mathbb{R}}|u-v|(T, x) d x \leqslant 2 \int_{0}^{T}\left|F^{1}-F^{2}\right|(t) d t+\int_{\mathbb{R}}\left|u_{0}-v_{0}\right|(x) d x .
$$

The proof of Proposition 2.10 is postponed to Appendix.

Combining the estimates (24) and (25) with a truncature and a density argument, we easily deduce the following generalization of Theorem 2.3

Theorem 2.11. For any $u_{0} \in \mathbf{L}^{\infty}(\mathbb{R} ;[0,1])$ and $F \in \mathbf{L}^{\infty}\left(\mathbb{R}^{+} ;[0, f(\bar{u})]\right)$ there exists one and only one CG-entropy solution to Problem 113) (which is also the unique $\mathcal{G}$-entropy solution of the problem).

Proof. The uniqueness claim is contained in Proposition 2.10 Let us prove the existence. Take the truncations $u_{0}(x) 1_{\{|x|<n\}}, F(t) 1_{\{0<t<n\}}$ and regularize them by convolution with a standard sequence of mollifiers $\left(\rho_{n}\right)_{n \in \mathbb{N}}$. Denote by $u_{0}^{n}, F^{n}$ the data obtained in this way; these data are of bounded variation. By Theorem 2.3 there exists a unique CG-entropy solution $u^{n}$ of (11) with the datum $u_{0}^{n}$ and the constraint $F^{n}$. By Theorem [2.9] $u^{n}$ is also a $\mathcal{G}$-entropy solution; in particular, it verifies the formulation (C) of Proposition 2.6

Clearly,

$$
u_{0}^{n} \rightarrow u_{0} \text { in } \mathbf{L}_{l o c}^{1}(\mathbb{R}) \text { and a.e.; } \quad F^{n} \rightarrow F \text { in } \mathbf{L}_{l o c}^{1}\left(\mathbb{R}^{+}\right) \text {and a.e.. }
$$


Combining (24) and (25) with (26), we infer that the sequence $\left(u^{n}\right)_{n \in \mathbb{N}}$ is a Cauchy sequence in $\mathbf{L}_{l o c}^{1}(\Pi)$. Further, notice that for all $\left(c_{l}, c_{r}\right) \in[0,1]^{2}$, for a.e. $t>0$ we have

$$
\operatorname{dist}\left(\left(c_{l}, c_{r}\right), \mathcal{G}\left(F^{n}(t)\right)\right) \longrightarrow \operatorname{dist}\left(\left(c_{l}, c_{r}\right), \mathcal{G}(F(t))\right) \text { as } n \rightarrow+\infty \text {. }
$$

Indeed, this follows from the explicit description of $\mathcal{G}(F)$ in Definition 2.4 from (26) and from the continuity of the map $F \mapsto\left(A_{F}, B_{F}\right)$, which stems from the continuity of the two branches of $f^{-1}$ :

$$
f_{-}^{-1}:[0, f(\bar{u})] \longrightarrow[0, \bar{u}] \text { and } f_{+}^{-1}:[0, f(\bar{u})] \longrightarrow[\bar{u}, 1] .
$$

Passing to the limit in the "global" entropy formulation (19) written for $u^{n}$, we infer that the $\mathbf{L}_{l o c}^{1}$ limit $u$ of $\left(u^{n}\right)_{n \in \mathbb{N}}$ is a $\mathcal{G}$-entropy solution of (13) associated with $u_{0}$ and $F$.

\section{$3 \quad$ Entropy process solutions}

We now look at more general solutions, that are entropy process solutions. They are based on an $\mathbf{L}^{\infty}$ representation of Young measures via their distribution function (see Eymard, Gallouët and Herbin [EGH00]; cf. Panov [DiP85, Sze89, Pan93). Entropy process solutions are very useful since they are a natural tool to investigate the limit of numerical schemes for which enough compactness (we mean in particular $B V$ bounds) cannot be proved. Besides, when the initial data is a usual initial condition $u_{0} \in \mathbf{L}^{\infty}(\mathbb{R})$, entropy process solutions coincide with entropy solutions; this is the reduction principle for entropy-process solutions. The reduction principle guarantees the convergence (in the strong a.e. sense) of the discrete solutions, obtained by numerical schemes, to the unique entropy solution.

Notice that it is difficult to generalize definition (A) of $\mathcal{G}$-entropy solutions, because the statement $\left(A_{2}\right)$ requires the existence of strong one-sided traces $\gamma^{l, r} u$ of $u$ on the interface $\{x=0\}$. In the case of entropy process solutions, only weak traces of the mean entropy fluxes $\int_{0}^{1} \Phi(\mu(\cdot, \alpha), \kappa) d \alpha$ are clearly available; fortunately, definition (B) can be recast in terms of these weak traces, whereas the "traceless" definition (C) is adapted in a straightforward way.

Proposition 3.1. Let $u_{0} \in \mathbf{L}^{\infty}(\mathbb{R},[0,1])$ and $F \in \mathbf{L}^{\infty}\left(\mathbb{R}^{+},[0, f(\bar{u})]\right)$. Let $\mu \in$ $\mathbf{L}^{\infty}(\Pi \times(0,1) ;[0,1])$. Then the assertions $(\mathbf{D})$ and $(\mathbf{E})$ below are equivalent:

(D) $\left(D_{1}\right) \mu$ is a Kruzhkov entropy process solution for $x<0$ and $x>0$ : for all nonnegative test functions $\varphi \in \mathcal{C}_{c}^{\infty}(\Pi \backslash\{x=0\})$ and all $\kappa \in[0,1]$,

$$
\begin{array}{r}
\int_{0}^{1} \int_{0}^{+\infty} \int_{\mathbb{R}}\left(|\mu(t, x, \alpha)-\kappa| \partial_{t}+\Phi(\mu(t, x, \alpha), \kappa) \partial_{x}\right) \varphi(t, x) d x d t d \alpha \\
+\int_{\mathbb{R}}\left|u_{0}(x)-\kappa\right| \varphi(0, x) d x \geqslant 0 ; \quad \text { (27) }
\end{array}
$$


$\left(D_{2}\right) \mu$ is a weak process solution: for all test functions $\varphi \in \mathcal{C}_{c}^{\infty}(\Pi)$,

$$
\begin{array}{r}
\int_{0}^{1} \int_{0}^{+\infty} \int_{\mathbb{R}}\left(\mu(t, x, \alpha) \partial_{t}+f(\mu(t, x, \alpha)) \partial_{x}\right) \varphi(t, x) d x d t d \alpha \\
+\int_{\mathbb{R}} u_{0}(x) \varphi(0, x) d x=0
\end{array}
$$

$\left(D_{3}\right)$ in addition, the weak traces $\left(\gamma_{w}^{l, r} \int_{0}^{1} \Phi\left(\mu(\cdot, \alpha), c_{l, r}\right) d \alpha\right)(t)$ satisfy for a.e. $t>0$ the inequalities

$$
\begin{aligned}
\forall\left(c_{l}, c_{r}\right) \in \mathcal{G}(F(t)) \quad \gamma_{w}^{l}\left[\int_{0}^{1} \Phi\left(\mu(\cdot, \alpha), c_{l}\right) d \alpha\right](t) \\
\geqslant \gamma_{w}^{r}\left[\int_{0}^{1} \Phi\left(\mu(\cdot, \alpha), c_{r}\right) d \alpha\right](t)
\end{aligned}
$$

(E) $\mu$ satisfies the following "global" entropy inequalities:

$\left(E_{1}\right)$ there exists $M>0$ such that for all $\left(c_{l}, c_{r}\right) \in[0,1]^{2}$ and all nonnegative test functions $\varphi \in \mathcal{C}_{c}^{\infty}(\Pi)$,

$$
\begin{aligned}
\int_{0}^{+\infty} \int_{\mathbb{R}} \int_{0}^{1}(\mid \mu(t, x, \alpha)- & \left.c(x) \mid \partial_{t}+\Phi(\mu(t, x, \alpha), c(x)) \partial_{x}\right) \varphi(t, x) d x d t d \alpha \\
& +\int_{\mathbb{R}}\left|u_{0}(x)-c(x)\right| \varphi(0, x) d x \\
\geqslant & -M \int_{0}^{+\infty} \operatorname{dist}\left(\left(c_{l}, c_{r}\right), \mathcal{G}(F(t))\right) \varphi(t, 0) d t, \quad(30)
\end{aligned}
$$

where $c(x)$ is the piecewise constant function given by (14), and dist refers to a distance function on $\mathbb{R}^{2}$.

Remark 6. Note that the existence of weak traces in $\left(D_{3}\right)$ follows from the entropy inequalities (27) and the boundedness of $\Phi(\mu, \kappa)$ (see Chen and Frid [CF99]). Let us precise the meaning of $\left(D_{3}\right)$. Since $\Phi$ is uniformly continuous on $[0,1]^{2}$, the traces $\gamma^{l, r}\left[\int_{0}^{1} \Phi(\mu(\cdot, \alpha), \kappa) d \alpha\right]$ depend continuously on $\kappa$ in the norm of $\mathbf{L}^{\infty}((0,+\infty))$. Therefore we can define these traces as maps

$$
\gamma^{l, r}: t \in(0,+\infty) \mapsto\left\{\kappa \mapsto\left(\gamma_{w}^{l, r} \int_{0}^{1} \Phi(\mu(\cdot, \alpha), \kappa) d \alpha\right)(t)\right\} \in C([0,1]) .
$$

Then (29) makes sense for all $t$ which is a common Lebesgue point of the above map and of the function $F$.

The proof of Proposition [3.1 is postponed to Appendix.

Definition 3.2. If any of the properties (D), (E) of Proposition 3.1]holds, then $\mu$ is called a $\mathcal{G}$-entropy process solution of Problem (113).

As mentioned in the Introduction, it is not clear whether $\mathscr{G}$-entropy process solutions are "intrinsically" unique. Indeed, we lack an explicit description, of 
the kind $\left(A_{2}\right)$, for the traces of $\mathcal{G}$-entropy process solution. This prevents us from mimicking the proof of uniqueness of entropy solutions; as a matter of fact, we are unable to give a sign to the term coming from the comparison of two $\mathcal{G}$-entropy process solutions at the interface $\{x=0\}$ (note that in BV06, Bachmann and Vovelle propose to use an even weaker notion of solution, based on a kinetic interpretation of the problem; it could be interesting to extend their idea to our framework). However, because we know the existence of a $\mathscr{G}$-entropy solution, we can compare a $\mathscr{G}$-entropy process solution with a $\mathscr{G}$ entropy solution and thus deduce the uniqueness and the reduction principle for $\mathcal{G}$-entropy solutions:

Proposition 3.3. Let $u_{0} \in \mathbf{L}^{\infty}(\mathbb{R} ;[0,1])$. If $u$ is the $\mathscr{G}$-entropy solution and if $\mu$ is a $\mathscr{G}$-entropy process solution, both associated with the same initial data $u_{0}$ and the same constraint $F$, then they coincide almost everywhere, i.e.,

$$
\mu(t, x, \alpha)=u(t, x) \quad \text { for a.e. } \alpha \in(0,1) \text { and a.e. }(t, x) \in \Pi .
$$

Proof. The proof is similar to the proof of Proposition 2.10] Considering nonnegative test functions $\varphi \in \mathcal{C}_{c}^{\infty}(\Pi \backslash\{x=0\})$, we obtain

$$
\begin{aligned}
\int_{0}^{1} \int_{\mathbb{R}^{+}} \int_{\mathbb{R} \backslash\{0\}}(\mid \mu(t, x, \alpha)- & u(t, x) \mid \partial_{t} \\
& \left.+\Phi(\mu(t, x, \alpha), u(t, x)) \partial_{x}\right) \varphi(t, x) d x d t d \alpha \geqslant 0 .
\end{aligned}
$$

Still choosing $\varphi$ as a sequence of approximations of the characteristic function of the set $\{t \in(0, T), 0<|x|<R+L(T-t)\}$ with $R, T>0$, from the previous inequality we deduce that

$$
\begin{array}{r}
-\int_{0}^{1} \int_{-R}^{R}|\mu(T, x, \alpha)-u(T, x)| d x d \alpha+\int_{0}^{T}\left(\gamma_{w}^{r}\left[\int_{0}^{1} \Phi(\mu(\cdot, \alpha), u(\cdot)) d \alpha\right]\right)(t) d t \\
-\int_{0}^{T}\left(\gamma_{w}^{l}\left[\int_{0}^{1} \Phi(\mu(\cdot, \alpha), u(\cdot)) d \alpha\right]\right)(t) d t \geqslant 0 ; \quad(32)
\end{array}
$$

the existence of the above weak traces follows from the previous inequality in the way of CF99. Moreover, the traces $\gamma^{l, r} u$ being strong, we have the identification

$$
\left.\gamma_{w}^{l, r}\left[\int_{0}^{1} \Phi(\mu(\cdot, \alpha), u(\cdot)) d \alpha\right] \equiv \gamma_{w}^{r}\left[\int_{0}^{1} \Phi(\mu(\cdot, \alpha), \kappa) d \alpha\right]\right|_{\kappa=\gamma^{l, r} u},
$$

where the weak traces in the right-hand side are those of Remark [6 Because $\left(\gamma^{l} u(t), \gamma^{r} u(t)\right) \in \mathscr{G}(F(t))$ we can apply inequality (29) of definition (D) of $\mathcal{G}$-entropy process solution. In this way, inequality (32) yields

$$
\text { for all } R>0 \text { and for a.e. } T>0 \quad \int_{0}^{1} \int_{-R}^{R}|\mu(T, x, \alpha)-u(T, x)| d x d \alpha \leqslant 0,
$$

which leads to the expected result (31). 


\section{Finite volume schemes}

We now turn on the construction of finite volume scheme to approximate the constrained problem (13). The aim is to develop a well-adapted (i.e., convergent and easy-to-implement!) numerical scheme starting from a monotone numerical flux.

\subsection{Construction of constrained schemes}

Let us first define the mesh.

Definition 4.1. An admissible mesh $\mathscr{T}$ of $\mathbb{R}$ is given by an increasing sequence of real values $\left(x_{i+1 / 2}\right)_{i \in \mathbb{Z}}$, such that $\mathbb{R}=\cup_{i \in \mathbb{Z}}\left[x_{i-1 / 2}, x_{i+1 / 2}\right]$ and $x_{1 / 2}=0$. The mesh $\mathscr{T}$ is the set $\mathscr{T}=\left\{K_{i}, i \in \mathbb{Z}\right\}$ of subsets of $\mathbb{R}$ defined by $K_{i}=$ $\left(x_{i-1 / 2}, x_{i+1 / 2}\right)$ for all $i \in \mathbb{Z}$. The length of $K_{i}$ is denoted by $h_{i}$ (the so-called space step), so that $h_{i}=x_{i+1 / 2}-x_{i-1 / 2}$ for all $i \in \mathbb{Z}$. We assume that the $h=\operatorname{size}(\mathscr{T})=\sup _{i \in \mathbb{Z}} h_{i}$ is finite and that, for some $\alpha \in \mathbb{R}_{+}^{*}, \alpha h \leqslant \inf _{i \in \mathbb{Z}} h_{i}$.

The finite volume approximation of the initial datum $u_{0}$ is

$$
u_{i}^{0}=\frac{1}{h_{i}} \int_{K_{i}} u_{0}(x) d x, \quad i \in \mathbb{Z} .
$$

We aim at defining a sequence $\left(u_{i}^{n}\right)_{i \in \mathbb{Z}, n \in \mathbb{N}}$ which approximates the solution $u$ of Problem (13) in the sense

$$
u_{i}^{n} \approx \frac{1}{h_{i}} \int_{K_{i}} u(n k, x) d x, \quad i \in \mathbb{Z}, n>0,
$$

where the time step $k$ is a positive constant (which will be prone to a CFL condition in the sequel). The finite volume scheme which is studied in the present work can be written under the form

$$
u_{i}^{n+1}=u_{i}^{n}-\lambda_{i}\left(g\left(u_{i}^{n}, u_{i+1}^{n}, F_{i+1 / 2}^{n}\right)-g\left(u_{i-1}^{n}, u_{i}^{n}, F_{i-1 / 2}^{n}\right)\right)
$$

where $\lambda_{i}=k / h_{i}$. The sequence $\left(F_{i+1 / 2}^{n}\right)_{i}$ is given by

$$
F_{i+1 / 2}^{n}= \begin{cases}(1 / \Delta t) \int_{n k}^{(n+1) k} F(s) d s & \text { if } i=0 \\ f(\bar{u}) & \text { if } i \neq 1 .\end{cases}
$$

Note that any approximation of $F$ which strongly converges in $\mathbf{L}_{\text {loc }}^{1}$ can be chosen to define $F_{1 / 2}^{n}$.

The numerical flux $g$ is defined by

$$
g(u, v, f)=\min (h(u, v), f),
$$

where $h$ is a classical numerical flux, i.e. it obeys the three classical properties:

- Regularity: $h$ is Lipschitz continuous, with $L$ as Lipschitz constant.

- Consistency: $h(s, s)=f(s)$ for any $s \in[0,1]$.

- Monotonicity: $h$ is nondecreasing with respect to (w.r.t.) its first argument and nonincreasing w.r.t. its second argument. 
We refer to Eymard, Gallouët and Herbin EGH00 for the background and examples of classical numerical fluxes.

We will also employ the notation

$$
u_{i}^{n+1}=\mathscr{G}_{\lambda_{i}}\left(u_{i-1}^{n}, u_{i}^{n}, u_{i+1}^{n}, F_{i-1 / 2}^{n}, F_{i+1 / 2}^{n}\right) .
$$

Let us now study this class of finite volume schemes.

\subsection{A priori estimates and discrete entropy inequalities}

We first prove the classical $\mathbf{L}^{\infty}$ estimate.

Proposition 4.2. Assume that $u_{0} \in \mathbf{L}^{\infty}(\mathbb{R} ;[0,1])$. Then, under the CFL condition

$$
k \leqslant \frac{\inf _{i} h_{i}}{2 L}
$$

the functions $\mathscr{G}_{\lambda_{i}}$ are nondecreasing w.r.t. their three first arguments and the finite volume approximation (34) satisfies

$$
0 \leqslant u_{i}^{n} \leqslant 1, \quad \forall n \in \mathbb{N}, \forall i \in \mathbb{Z} .
$$

Proof. Let us successively derive the function $\mathscr{G}_{\lambda}$ w.r.t. its three first arguments:

$$
\begin{aligned}
& \partial_{1} \mathscr{G}_{\lambda}\left(u, v, w, F_{1}, F_{2}\right)=\lambda \partial_{1} g\left(u, v, F_{1}\right), \\
& \partial_{2} \mathscr{G}_{\lambda}\left(u, v, w, F_{1}, F_{2}\right)=1-\lambda\left(\partial_{1} g\left(v, w, F_{2}\right)-\partial_{2} g\left(u, v, F_{1}\right)\right), \\
& \partial_{3} \mathscr{G}_{\lambda}\left(u, v, w, F_{1}, F_{2}\right)=-\lambda \partial_{2} g\left(v, w, F_{2}\right) .
\end{aligned}
$$

By monotonicity of $h$, the first and the last partial derivates are nonnegative. The second partial derivate is nonnegative under condition (38).

Using the consistency of the flux $h$, we have

$$
\mathscr{G}_{\lambda}\left(0,0,0, F_{1}, F_{2}\right)=-\lambda\left(\min \left(f(0), F_{2}\right)-\min \left(f(0), F_{1}\right)\right)=0
$$

and

$$
\mathscr{G}_{\lambda}\left(1,1,1, F_{1}, F_{2}\right)=1-\lambda\left(\min \left(f(1), F_{2}\right)-\min \left(f(1), F_{1}\right)\right)=1 .
$$

Therefore, if $\left(u_{i}^{n}\right)_{i}$ lies in $[0,1]$, then $\left(u_{i}^{n+1}\right)_{i}$ also belongs to [0,1] by monotonicity of $\mathscr{G}_{\lambda}$. This leads to the maximum principle (39).

We now focus on the so-called weak- $B V$ estimate (cf. [EGH00]). In the sequel, we adopt the notations: $a \perp b=\min (a, b)$ and $a \top b=\max (a, b)$.

Lemma 4.3. Let $\xi \in(0,1)$ and $\mathscr{T}$ be an admissible mesh. Let $T>k$ and $R>h$ be two positive constants and denote $I_{0}, I_{1}$ and $N$ the indices such that $-R \in \bar{K}_{I_{0}}, R \in \bar{K}_{I_{1}}$ and $T \in(N k,(N+1) k]$. Then, if the time step $k$ satisfies the CFL condition

$$
k \leqslant(1-\xi) \frac{\inf _{i \in \mathbb{Z}} h_{i}}{2 L}
$$

there exists a positive constant $C$ only depending on $T, R, \xi, \alpha, f$ and $u_{0}$ such that

$$
\begin{aligned}
k \sum_{n=0}^{N} \sum_{\substack{i=I_{0} \\
i \neq 0,1}}^{I_{1}}\left(\max _{(p, q) \in \mathbb{I}\left(u_{i}^{n}, u_{i+1}^{n}\right)}|h(p, q)-f(p)|\right. & \\
& \left.\quad+\max _{(p, q) \in \mathbb{I}\left(u_{i}^{n}, u_{i+1}^{n}\right)}|h(p, q)-f(q)|\right) \leqslant C h^{-1 / 2},
\end{aligned}
$$

where the set $\mathbb{I}(a, b)$ is defined as $\{(p, q) \in[a \perp b, a \top b],(q-p)(b-a) \geqslant 0\}$. 
Proof. Since all the terms of the sum in (41) are nonnegative, we have

$$
\begin{aligned}
& k \sum_{n=0}^{N} \sum_{\substack{i=I_{0} \\
i \neq 0,1}}^{I_{1}}\left(\max _{(p, q) \in \mathbb{I}\left(u_{i}^{n}, u_{i+1}^{n}\right)}|h(p, q)-f(p)|+\max _{(p, q) \in \mathbb{I}\left(u_{i}^{n}, u_{i+1}^{n}\right)}|h(p, q)-f(q)|\right) \\
\leqslant & k \sum_{n=0}^{N} \sum_{i=I_{0}}^{I_{1}}\left(\max _{(p, q) \in \mathbb{I}\left(u_{i}^{n}, u_{i+1}^{n}\right)}|h(p, q)-f(p)|+\max _{(p, q) \in \mathbb{I}\left(u_{i}^{n}, u_{i+1}^{n}\right)}|h(p, q)-f(q)|\right) .
\end{aligned}
$$

The right-hand side of the previous inequality is classical, since this term appears when dealing with a scalar conservation law approximated by a classical finite volume monotone scheme. Following [EGH00] for instance, we see that it admits $C h^{-1 / 2}$ as an upper-bound; this yields (41).

The two previous estimates will enable us to pass to the limit, using the nonlinear weak- $\star$ convergence (see Definition 4.5 below). In order to show that the limit is a $\mathcal{G}$-entropy process solution of Problem (13), we derive discrete entropy inequalities. We have:

Proposition 4.4. Let $\kappa_{i}=c_{l}$ for $i \leqslant 0$ and $\kappa_{i}=c_{r}$ for $i>0$, where $\left(c_{l}, c_{r}\right) \in$ $[0,1]^{2}$. Then, the numerical scheme (34)-(36) fulfills the following inequalities:

$$
\left|u_{i}^{n+1}-\kappa_{i}\right|-\left|u_{i}^{n}-\kappa_{i}\right|+\lambda_{i}\left(G_{i+1 / 2}^{n}-G_{i-1 / 2}^{n}\right)-\lambda_{i}\left|H_{i}^{n}\right| \leqslant 0
$$

where

$$
\begin{aligned}
G_{i+1 / 2}^{n} & =g\left(u_{i}^{n} \top \kappa_{i}, u_{i+1}^{n} \top \kappa_{i+1}, F_{i+1 / 2}\right)-g\left(u_{i}^{n} \perp \kappa_{i}, u_{i+1}^{n} \perp \kappa_{i+1}, F_{i+1 / 2}\right), \\
H_{i}^{n} & =h\left(\kappa_{i}, \kappa_{i+1}\right) \perp F_{i+1 / 2}^{n}-h\left(\kappa_{i-1}, \kappa_{i}\right) \perp F_{i-1 / 2}^{n},
\end{aligned}
$$

for all $n \in \mathbb{N}$ and $i \in \mathbb{Z}$.

Proof. Let us first note that

$$
\mathscr{G}_{\lambda_{i}}\left(\kappa_{i-1}, \kappa_{i}, \kappa_{i+1}, F_{i-1 / 2}^{n}, F_{i+1 / 2}^{n}\right)=\kappa_{i}-\lambda_{i} H_{i}^{n} .
$$

In order to prove inequality (42), two cases must be considered according to the sign of $H_{i}^{n}$. Assume first that $H_{i}^{n} \geqslant 0$. Let us recall that

$$
\left|u_{i}^{n+1}-\kappa\right|=u_{i}^{n+1} \top \kappa-u_{i}^{n+1} \perp \kappa
$$

and estimate the two terms of the right-hand side. We have for the first term

$$
\begin{aligned}
u_{i}^{n+1} \top \kappa_{i}-\lambda_{i} H_{i}^{n}= & \left(u_{i}^{n+1}-\lambda_{i} H_{i}^{n}\right) \top\left(\kappa-\lambda_{i} H_{i}^{n}\right), \\
= & \left(u_{i}^{n+1}-\lambda_{i} H_{i}^{n}\right) \top \mathscr{G}_{\lambda_{i}}\left(\kappa_{i-1}, \kappa_{i}, \kappa_{i+1}, F_{i-1 / 2}^{n}, F_{i+1 / 2}^{n}\right), \\
\leqslant & u_{i}^{n+1} \top \mathscr{G}_{\lambda_{i}}\left(\kappa_{i-1}, \kappa_{i}, \kappa_{i+1}, F_{i-1 / 2}^{n}, F_{i+1 / 2}^{n}\right), \\
\leqslant & \mathscr{G}_{\lambda_{i}}\left(u_{i-1}^{n}, u_{i}^{n}, u_{i+1}^{n}, F_{i-1 / 2}^{n}, F_{i+1 / 2}^{n}\right) \\
& \top \mathscr{G}_{\lambda_{i}}\left(\kappa_{i-1}, \kappa_{i}, \kappa_{i+1}, F_{i-1 / 2}^{n}, F_{i+1 / 2}^{n}\right), \\
\leqslant & \mathscr{G}_{\lambda_{i}}\left(u_{i-1}^{n} \top \kappa_{i-1}, u_{i}^{n} \top \kappa_{i}, u_{i+1}^{n} \top \kappa_{i+1}, F_{i-1 / 2}^{n}, F_{i+1 / 2}^{n}\right) .
\end{aligned}
$$


For the second term, we have

$$
\begin{aligned}
u_{i}^{n+1} \perp \kappa_{i}= & \mathscr{G}_{\lambda_{i}}\left(u_{i-1}^{n}, u_{i}^{n}, u_{i+1}^{n}, F_{i-1 / 2}^{n}, F_{i+1 / 2}^{n}\right) \perp \kappa_{i}, \\
\geqslant & \mathscr{G}_{\lambda_{i}}\left(u_{i-1}^{n}, u_{i}^{n}, u_{i+1}^{n}, F_{i-1 / 2}^{n}, F_{i+1 / 2}^{n}\right) \perp\left(\kappa_{i}-\lambda_{i} H_{i}^{n}\right), \\
\geqslant & \mathscr{G}_{\lambda_{i}}\left(u_{i-1}^{n}, u_{i}^{n}, u_{i+1}^{n}, F_{i-1 / 2}^{n}, F_{i+1 / 2}^{n}\right) \\
& \perp \mathscr{G}_{\lambda_{i}}\left(\kappa_{i-1}, \kappa_{i}, \kappa_{i+1}, F_{i-1 / 2}^{n}, F_{i+1 / 2}^{n}\right), \\
\geqslant & \mathscr{G}_{\lambda_{i}}\left(u_{i-1}^{n} \perp \kappa_{i-1}, u_{i}^{n} \perp \kappa_{i}, u_{i+1}^{n} \perp \kappa_{i+1}, F_{i-1 / 2}^{n}, F_{i+1 / 2}^{n}\right) .
\end{aligned}
$$

Therefore, gathering the two latter estimates yields

$$
\begin{aligned}
\left|u_{i}^{n+1}-\kappa_{i}\right|= & u_{i}^{n+1} \top \kappa_{i}-u_{i}^{n+1} \perp \kappa_{i}, \\
\leqslant & \lambda_{i} H_{i}^{n}+\mathscr{G}_{\lambda_{i}}\left(u_{i-1}^{n} \top \kappa_{i-1}, u_{i}^{n} \top \kappa_{i}, u_{i+1}^{n} \top \kappa_{i+1}, F_{i-1 / 2}^{n}, F_{i+1 / 2}^{n}\right) \\
& -\mathscr{G}_{\lambda_{i}}\left(u_{i-1}^{n} \perp \kappa_{i-1}, u_{i}^{n} \perp \kappa_{i}, u_{i+1}^{n} \perp \kappa_{i+1}, F_{i-1 / 2}^{n}, F_{i+1 / 2}^{n}\right) .
\end{aligned}
$$

This inequality, by definition of $\mathscr{G}_{\lambda_{i}}$, becomes

$$
\begin{aligned}
& \left|u_{i}^{n+1}-\kappa_{i}\right| \leqslant u_{i}^{n} \top \kappa_{i}-u_{i}^{n} \perp \kappa_{i}+\lambda_{i} H_{i}^{n} \\
& \quad-\lambda_{i}\left(g\left(u_{i}^{n} \top \kappa_{i}, u_{i+1}^{n} \top \kappa_{i+1}, F_{i+1 / 2}^{n}\right)-g\left(u_{i-1}^{n} \top \kappa_{i-1}, u_{i}^{n} \top \kappa_{i}, F_{i-1 / 2}^{n}\right)\right) \\
& \quad+\lambda_{i}\left(g\left(u_{i}^{n} \perp \kappa_{i}, u_{i+1}^{n} \perp \kappa_{i+1}, F_{i+1 / 2}^{n}\right)-g\left(u_{i-1}^{n} \perp \kappa_{i-1}, u_{i}^{n} \perp \kappa_{i}, F_{i-1 / 2}^{n}\right)\right),
\end{aligned}
$$

which is exactly inequality [42). The case $H_{i}^{n} \leqslant 0$ is analogous.

\subsection{Approximate solutions and convergence}

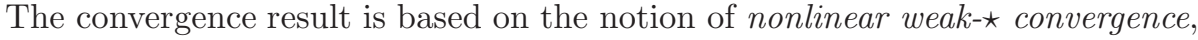
defined in EGH00:

Definition 4.5. Let $\Omega$ be an open subset of $\mathbb{R}^{N}, N \geqslant 1,\left(u_{m}\right)_{m \in \mathbb{N}} \subset \mathbf{L}^{\infty}(\Omega)$ and $\mu \in \mathbf{L}^{\infty}(\Omega \times(0,1))$. The sequence $\left(u_{m}\right)_{m \in \mathbb{N}}$ converges to $\mu$ in the nonlinear weak $-\star$ sense if

$$
\int_{\Omega} \theta\left(u_{m}(y)\right) \varphi(y) d y \underset{m \rightarrow \infty}{\longrightarrow} \int_{\Omega} \int_{0}^{1} \theta(\mu(y, \alpha)) \varphi(y) d y d \alpha
$$

for all $\varphi \in \mathbf{L}^{1}(\Omega)$ and all $\theta \in \mathcal{C}(\Omega)$.

This definition provides a useful interpretation of the convergence to Young's measures (as used by DiPerna DiP85] and Szepessy [Sze89]). It enables to obtain the convergence of any sequence (up to a subsequence) just using a $\mathbf{L}^{\infty}(\Omega)$ bound:

Theorem 4.6. Let $\Omega$ be an open subset of $\mathbb{R}^{N}, N \geqslant 1$. Consider a bounded sequence $\left(u_{m}\right)_{m \in \mathbb{N}}$ in $\mathbf{L}^{\infty}(\Omega)$. Then, one can extract a subsequence to $\left(u_{m}\right)_{m \in \mathbb{N}}$

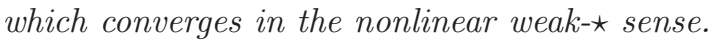

Moreover, the convergence is strong (in the $\mathbf{L}_{\text {loc }}^{1}(\Omega)$ sense) if and only if the nonlinear weak- $\star$ limit $\mu$ of $\left(u_{m}\right)_{m \in \mathbb{N}}$ is independent of $\alpha$.

By Definition 4.5 in the situation of Theorem 4.6 weakly in $\mathbf{L}_{l o c}^{1}(\Omega)$ to $\int_{0}^{1} \theta(\mu(., \alpha)) d \alpha$, for all $\theta \in \mathcal{C}(\mathbb{R})$. In the sequel, we will not re-label the subsequences; the uniqueness of a $\mathcal{G}$-entropy solution will ensure that all subsequences 
converge to the same limit, and the reduction of $\mu$ to an $\alpha$-independent function will ensure that the convergence is strong.

Now, let us define the sequence of approximate solutions:

$$
u_{\mathscr{T}, k}=u_{i}^{n} \quad \text { for } x \in K_{i} \text { and } t \in[n k,(n+1) k) .
$$

where $\left(u_{i}^{n}\right)_{i \in \mathbb{Z}, n \in \mathbb{N}}$ is defined by the numerical scheme (34 36). Existence of the approximate solutions is trivial. Thanks to the a priori bounds and to the discrete entropy inequalities of Section 4.2 we can prove the following convergence result:

Proposition 4.7. Let $\xi, \alpha \in(0,1)$. Consider a sequence of admissible meshes $\mathscr{T}_{m}$ and of time steps $k_{m}$ satisfying the stability condition (38) for all $m \in \mathbb{N}$, such that $\operatorname{size}\left(\mathscr{T}_{m}\right) \rightarrow 0$ as $m \rightarrow \infty$.

Consider the sequence $\left(u_{\mathscr{T}_{m}, k_{m}}\right)_{m \in \mathbb{N}}$, which is bounded in $\mathbf{L}^{\infty}(\Pi)$. Then, there exists a subsequence, still noted $\left(u_{\mathscr{T}_{m}, k_{m}}\right)_{m \in \mathbb{N}}$, and a function $\mu \in \mathbf{L}^{\infty}(\Pi \times$ $(0,1))$ such that $\left(u_{\mathscr{T}_{m}, k_{m}}\right)_{m}$ tends to $\mu$ in the nonlinear weak- $\star$ sense as $m \rightarrow$ $+\infty$, and $\mu$ satisfies

$$
\begin{gathered}
\int_{0}^{1} \int_{0}^{+\infty} \int_{\mathbb{R}}\left(|\mu(t, x, \alpha)-c(x)| \partial_{t}+\Phi(\mu(t, x, \alpha), c(x)) \partial_{x}\right) \varphi(t, x) d x d t d \alpha \\
\quad+\int_{\mathbb{R}}\left|u_{0}(x)-c(x)\right| \varphi(0, x) d x \\
+12 L \int_{0}^{+\infty} \operatorname{dist}\left(\left(c_{l}, c_{r}\right), \mathscr{G}_{1}(F(t)) \cup \mathscr{G}_{2}(F(t))\right) \varphi(t, 0) d t \geqslant 0,
\end{gathered}
$$

for all nonnegative test function $\varphi \in \mathcal{C}_{c}^{\infty}(\Pi)$ and all function $c(x)$ given by (14) with $\left(c_{l}, c_{r}\right) \in[0,1]^{2}$.

Notice that inequality (45) seems weaker than the inequalities (30) in the definition of $\mathcal{G}$-entropy process solutions. In fact, it follows from Lemma 4.8 below that the two families of inequalities are equivalent.

Proof. The convergence of the subsequence $\left(u_{\mathscr{T}_{m}, k_{m}}\right)_{m}$ to $\mu$ follows by Proposition 4.2 and Theorem 4.6. We must now prove that $\mu$ satisfies 45 .

Let $\varphi \in \mathcal{C}_{c}^{\infty}(\Pi)$ nonnegative and two positive constants, $T$ and $R$, such that for all $t \geqslant T$ and $|x| \geqslant R, \varphi(t, x)=0$ (we choose $T$ and $R$ sufficiently large w.r.t. $h$ and $k$ ). Besides, let $I_{0}, I_{1}$ and $N$ be the indices satisfying $-R \in \bar{K}_{I_{0}}$, $R \in \bar{K}_{I_{1}}$ and $T \in(N k,(N+1) k]$.

We multiply the discrete entropy inequality (42) by $\int_{K_{i}} \varphi(n k, x) d x$ and sum for $n \in[0, N]$ and $i \in\left[I_{0}, I_{1}\right]$, which yields

$$
A_{h}+B_{h}+C_{h} \leqslant 0
$$


where

$$
\begin{aligned}
A_{h} & =\sum_{n=0}^{N} \sum_{i=I_{0}}^{I_{1}}\left(\left|u_{i}^{n+1}-\kappa_{i}\right|-\left|u_{i}^{n}-\kappa_{i}\right|\right) \int_{K_{i}} \varphi(n k, x) d x \\
B_{h} & =\sum_{n=0}^{N} \sum_{i=I_{0}}^{I_{1}} \lambda_{i}\left(G_{i+1 / 2}^{n}-G_{i-1 / 2}^{n}\right) \int_{K_{i}} \varphi(n k, x) d x \\
C_{h} & =-\sum_{n=0}^{N} \sum_{i=I_{0}}^{I_{1}} \lambda_{i}\left|H_{i}^{n}\right| \int_{K_{i}} \varphi(n k, x) d x
\end{aligned}
$$

We aim at passing to the limit $h \rightarrow 0$ in (46) and recover the definition of the $\mathscr{G}$-entropy process solution. The convergence of the term $A_{h}$ is achieved using the Abel transform:

$$
\begin{aligned}
A_{h}= & -\sum_{n=1}^{N+1} \sum_{i=I_{0}}^{I_{1}}\left|u_{i}^{n}-\kappa_{i}\right| \int_{K_{i}}(\varphi(n k, x)-\varphi((n-1) k, x)) d x \\
& -\sum_{i=I_{0}}^{I_{1}}\left|u_{i}^{0}-\kappa_{i}\right| \int_{K_{i}} \varphi(0, x) d x,
\end{aligned}
$$

which, $u_{\mathscr{T}, k}$ being constant in $K_{i}$, leads to

$$
\begin{aligned}
A_{h}= & -\sum_{n=1}^{N+1} \sum_{i=I_{0}}^{I_{1}} \int_{K_{i}} \int_{n k}^{(n+1) k}\left|u_{\mathscr{T}, k}(t, x)-\kappa_{i}\right| \frac{\varphi(n k, x)-\varphi((n-1) k, x)}{k} d t d x \\
& -\sum_{i=I_{0}}^{I_{1}} \int_{K_{i}}\left|u_{\mathscr{T}, k}(0, x)-\kappa_{i}\right| \varphi(0, x) d x .
\end{aligned}
$$

Passing to the limit in the last expression (in the nonlinear weak- $\star$ sense for $\left|u_{\mathscr{T}, k}-\kappa(x)\right|$ and using the regularity of $\varphi$ ) gives

$$
\begin{aligned}
\lim _{h \rightarrow 0} A_{h}= & -\int_{0}^{1} \int_{0}^{\infty} \int_{\mathbb{R}}|\mu(t, x, \alpha)-\kappa(x)| \partial_{t} \varphi d x d t d \alpha \\
& -\int_{\mathbb{R}}\left|u_{0}(x)-\kappa(x)\right| \varphi(0, x) d x .
\end{aligned}
$$

These terms appear indeed in 45.

Let us now focus on $B_{h}$. Two difficulties arise in comparison with the computations done for $A_{h}$. The first one concerns the Abel transform. Since the spatial mesh is not uniform, this transform cannot be achieved directly. But this difficulty is classical and can be easily settled by decoupling the convergence of terms in $u_{m}$ and the convergence of terms in $\varphi$. The second difficulty is more specific to our problem: the numerical flux has an extra dependence in $F$ and $\kappa$ depends on the space variable. We will see that it does not alter the convergence of $B_{h}$, that is to say, we will obtain

$$
\lim _{h \rightarrow 0} B_{h}=-\int_{0}^{1} \int_{0}^{\infty} \int_{\mathbb{R}} \Phi(\mu(t, x, \alpha), \kappa(x)) \partial_{x} \varphi(t, x) d x d t d \alpha \quad(=: B) .
$$


We introduce

$$
B_{h}^{\prime}=-\sum_{n=0}^{N} \sum_{i=I_{0}}^{I_{1}} G_{i+1 / 2}^{n} \int_{K_{i}} \int_{n k}^{(n+1) k} \partial_{x} \varphi(t, x) d t d x
$$

and we will prove that

$$
\lim _{h \rightarrow 0}\left|B_{h}-B_{h}^{\prime}\right|=0 \quad \text { and } \quad \lim _{h \rightarrow 0}\left|B_{h}^{\prime}-B\right|=0 .
$$

We begin by studying the first limit. The term $B_{h}^{\prime}$ becomes

$$
\begin{aligned}
B_{h}^{\prime} & =-\sum_{n=0}^{N} \sum_{i=I_{0}}^{I_{1}} G_{i+1 / 2}^{n} \int_{n k}^{(n+1) k}\left(\varphi\left(t, x_{i+1 / 2}\right)-\varphi\left(t, x_{i-1 / 2}\right)\right) d t, \\
& =\sum_{n=0}^{N} \sum_{i=I_{0}}^{I_{1}}\left(G_{i+1 / 2}^{n}-G_{i-1 / 2}^{n}\right) \int_{n k}^{(n+1) k} \varphi\left(t, x_{i-1 / 2}\right) d t,
\end{aligned}
$$

using the Abel transform and the compact support of $\varphi$. We can now compare $B_{h}$ and $B_{h}^{\prime}$ :

$$
\begin{aligned}
\left|B_{h}-B_{h}^{\prime}\right|= & \mid \sum_{n=0}^{N} \sum_{i=I_{0}}^{I_{1}} \lambda_{i}\left(G_{i+1 / 2}^{n}-G_{i-1 / 2}^{n}\right) \int_{K_{i}} \varphi(n k, x) d x \\
& -\sum_{n=0}^{N} \sum_{i=I_{0}}^{I_{1}}\left(G_{i+1 / 2}^{n}-G_{i-1 / 2}^{n}\right) \int_{n k}^{(n+1) k} \varphi\left(t, x_{i-1 / 2}\right) d t \mid, \\
\leqslant & \sum_{n=0}^{N} \sum_{i=I_{0}}^{I_{1}}\left|G_{i+1 / 2}^{n}-G_{i-1 / 2}^{n}\right| \\
& \int_{n k}^{(n+1) k}\left|\frac{1}{h_{i}} \int_{K_{i}} \varphi(n k, x) d x-\varphi\left(t, x_{i-1 / 2}\right)\right| d t \\
\leqslant & \sum_{n=0}^{N} \sum_{i=I_{0}}^{I_{1}}\left|G_{i+1 / 2}^{n}-G_{i-1 / 2}^{n}\right| \\
& \int_{n k}^{(n+1) k} \frac{1}{h_{i}} \int_{K_{i}}\left|\varphi(n k, x) d x-\varphi\left(t, x_{i-1 / 2}\right)\right| d t .
\end{aligned}
$$

Since $\varphi$ belongs to $\mathcal{C}_{c}^{\infty}\left(\mathbb{R}_{+} \times \mathbb{R}\right)$, there exists a positive constant $C$ such that

$$
\left|B_{h}-B_{h}^{\prime}\right| \leqslant C k h \sum_{n=0}^{N} \sum_{i=I_{0}}^{I_{1}}\left|G_{i+1 / 2}^{n}-G_{i-1 / 2}^{n}\right| .
$$

In order to check that $\left|B_{h}-B_{h}^{\prime}\right|$ tends to 0 as $h \rightarrow 0$ the sum over $i$ is split in three parts: $i \in\{0,1\}, i \in\left[I_{0},-1\right]$ and $i \in\left[2, I_{1}\right]$. The first part becomes

$$
k h \sum_{n=0}^{N} \sum_{i=0,1}\left|G_{i+1 / 2}^{n}-G_{i-1 / 2}^{n}\right| \leqslant C h \sum_{n=0}^{N} k \leqslant C h T .
$$


Concerning the second and the third parts, since $i \neq 0,1$, then

$$
\begin{aligned}
\left|G_{i+1 / 2}^{n}-G_{i-1 / 2}^{n}\right|= & \mid h\left(u_{i}^{n} \top \kappa_{i}, u_{i+1}^{n} \top \kappa_{i+1}\right)-h\left(u_{i}^{n} \perp \kappa_{i}, u_{i+1}^{n} \perp \kappa_{i+1}\right) \\
& -h\left(u_{i-1}^{n} \top \kappa_{i-1}, u_{i}^{n} \top \kappa_{i}\right)+h\left(u_{i-1}^{n} \perp \kappa_{i-1}, u_{i}^{n} \perp \kappa_{i}\right) \mid .
\end{aligned}
$$

In order to use the weak- $B V$ estimate (41), we introduce in the last equality $f\left(u_{i}^{n} \perp \kappa_{i}\right)$ and $f\left(u_{i}^{n} \top \kappa_{i}\right)$, which gives

$$
\begin{aligned}
& \left|G_{i+1 / 2}^{n}-G_{i-1 / 2}^{n}\right| \\
& \quad \leqslant\left|h\left(u_{i}^{n} \top \kappa_{i}, u_{i+1}^{n} \top \kappa_{i+1}\right)-f\left(u_{i}^{n} \top \kappa_{i}\right)\right|+\left|h\left(u_{i}^{n} \perp \kappa_{i}, u_{i+1}^{n} \perp \kappa_{i+1}\right)-f\left(u_{i}^{n} \perp \kappa_{i}\right)\right| \\
& \quad+\left|h\left(u_{i-1}^{n} \top \kappa_{i-1}, u_{i}^{n} \top \kappa_{i}\right)-f\left(u_{i}^{n} \top \kappa_{i}\right)\right|+\left|h\left(u_{i-1}^{n} \perp \kappa_{i-1}, u_{i}^{n} \perp \kappa_{i}\right)-f\left(u_{i}^{n} \perp \kappa_{i}\right)\right| \\
& \quad \leqslant 2\left(\max _{(p, q) \in \mathbb{I}\left(u_{i}^{n}, u_{i+1}^{n}\right)}|h(p, q)-f(p)|+\max _{(p, q) \in \mathbb{I}\left(u_{i}^{n}, u_{i+1}^{n}\right)}|h(p, q)-f(q)|\right) .
\end{aligned}
$$

Therefore, Lemma 4.3 provides the estimate

$$
k h \sum_{n=0}^{N} \sum_{\substack{i=I_{0} \\ i \neq 0,1}}^{I_{1}}\left|G_{i+1 / 2}^{n}-G_{i-1 / 2}^{n}\right| \leqslant C \sqrt{h} .
$$

Then, using (50) and (51), we obtain the first limit of (49) and can now focus on the second limit. The term $B$ can be modified as

$$
\begin{aligned}
& B=-\sum_{n=0}^{N} \sum_{i=I_{0}}^{I_{1}} \int_{n k}^{(n+1) k} \int_{K_{i}} \int_{0}^{1}( f\left(\mu(t, x, \alpha) \top \kappa_{i}\right) \\
&\left.-f\left(\mu(t, x, \alpha) \perp \kappa_{i}\right)\right) \partial_{x} \varphi(t, x) d x d t d \alpha .
\end{aligned}
$$

The terms $B_{h}^{\prime}$ and $B$ can now be compared:

$$
\begin{aligned}
& \left|B_{h}^{\prime}-B\right| \leqslant \sum_{n=0}^{N} \sum_{i=I_{0}}^{I_{1}} \int_{K_{i}} \int_{n k}^{(n+1) k} \int_{0}^{1} \mid\left(f\left(\mu \top \kappa_{i}\right)-f\left(\mu \perp \kappa_{i}\right)\right) \\
& \text { - }\left(g\left(u_{i}^{n} \top \kappa_{i}, u_{i+1}^{n} \top \kappa_{i+1}, F_{i+1 / 2}\right)\right. \\
& \left.-g\left(u_{i}^{n} \perp \kappa_{i}, u_{i+1}^{n} \perp \kappa_{i+1}, F_{i+1 / 2}\right)\right)|| \partial_{x} \varphi \mid d \alpha d t d x
\end{aligned}
$$

The same difficulty as above occurs and the sum over $i$ must be split in three parts: $i=0, i \in\left[I_{0},-1\right]$ and $i \in\left[1, I_{1}\right]$. Since both functions $f$ and $g$ are Lipschitz continuous, the first $(i=0)$ can be easily handled:

$$
\begin{aligned}
& \sum_{n=0}^{N} \int_{K_{0}} \int_{n k}^{(n+1) k} \int_{0}^{1} \mid\left(g\left(u_{0}^{n} \top \kappa_{0}, u_{1}^{n} \top \kappa_{1}, F_{1 / 2}\right)-g\left(u_{0}^{n} \perp \kappa_{0}, u_{1}^{n} \perp \kappa_{1}, F_{1 / 2}\right)\right) \\
&-\left.\left(f\left(\mu \top \kappa_{i}\right)-f\left(\mu \perp \kappa_{i}\right)\right)|| \partial_{x} \varphi\left|d \alpha d t \leqslant C \| \partial_{x} \varphi\right|\right|_{\infty} T h
\end{aligned}
$$

For the two other sums, $i \in\left[I_{0},-1\right]$ and $i \in\left[1, I_{1}\right]$, the procedure is the same as done for $\left|B_{h}-B_{h}^{\prime}\right|$, using the weak- $B V$ estimate. It finally provides the second limit of (49) and thus (48). It remains to check that

$$
\lim _{h \rightarrow 0} C_{h}=-12 L \int_{0}^{\infty} \operatorname{dist}\left(\left(c_{l}, c_{r}\right), \mathscr{G}_{1}(F(t)) \cup \mathscr{G}_{2}(F(t))\right) \varphi(t, 0) d t .
$$


By definition of $H_{i}^{n}$, we obtain

$$
\begin{aligned}
C_{h}= & -\sum_{i=0}^{1} \frac{1}{h_{i}} \int_{K_{i}} \sum_{n=0}^{N}\left|H_{i}^{n}\right| k \varphi(n k, x) d x \\
= & -\frac{1}{h_{0}} \int_{K_{0}} \sum_{n=0}^{N}\left|h\left(c_{l}, c_{r}\right) \perp F^{n}-f\left(c_{l}\right)\right| k \varphi(n k, x) d x \\
& -\frac{1}{h_{1}} \int_{K_{1}} \sum_{n=0}^{N}\left|f\left(c_{r}\right)-h\left(c_{l}, c_{r}\right) \perp F^{n}\right| k \varphi(n k, x) d x .
\end{aligned}
$$

Since $\varphi$ is smooth and $F^{n}$ strongly converges to $F$, we obtain the limit

$$
\lim _{h \rightarrow 0} C_{h}=-2 \int_{0}^{\infty} D\left(c_{l}, c_{r}, F(t)\right) \varphi(t, 0) d t
$$

where

$$
D\left(c_{l}, c_{r}, F\right)=\left|h\left(c_{l}, c_{r}\right) \perp F-f\left(c_{l}\right)\right|+\left|f\left(c_{r}\right)-h\left(c_{l}, c_{r}\right) \perp F\right| .
$$

The function $D$ is Lipschitz continuous with respect to $c_{l}$ and $c_{r}$, with constant $6 L$. Moreover, if $\left(c_{l}, c_{r}\right) \in \mathscr{G}_{1}(F) \cup \mathscr{G}_{2}(F)$, then $D\left(c_{l}, c_{r}, F\right)=0$ (it is no longer true if $\left(c_{l}, c_{r}\right) \in \mathscr{G}_{3}(F)$ since a monotone scheme does not necessarily preserve stationary shock waves). Therefore

$$
D\left(c_{l}, c_{r}, F\right) \leqslant 6 L \operatorname{dist}\left(\left(c_{l}, c_{r}\right), \mathscr{G}_{1}(F) \cup \mathscr{G}_{2}(F)\right),
$$

so that the limit verifies the entropy inequalities 450.

It remains to prove that if inequalities (45) hold, then $\mu$ is a $\mathscr{G}$-entropy process solution. Indeed, we have the following lemma.

Lemma 4.8. Let $\eta \in \mathbf{L}^{\infty}(\Pi \times(0,1))$. Assume that the weak traces (in the sense of Remark G) $\gamma_{w}^{l, r} \int_{0}^{1} \Phi(\eta(\cdot, \alpha), \kappa)$ d $\alpha$ exist. If for a.e. $t>0$, the inequality

$$
\left(\gamma_{w}^{l} \int_{0}^{1} \Phi\left(\eta(\cdot, \alpha), c_{l}\right) d \alpha\right)(t) \geqslant\left(\gamma_{w}^{r} \int_{0}^{1} \Phi\left(\eta(\cdot, \alpha), c_{r}\right) d \alpha\right)(t)
$$

holds for all $\left(c_{l}, c_{r}\right) \in \mathscr{G}_{1}(F(t)) \cup \mathscr{G}_{2}(F(t))$, then it also holds for all $\left(c_{l}, c_{r}\right) \in$ $\mathscr{G}_{3}(F(t))$.

Proof. Let $\left(c_{l}, c_{r}\right) \in \mathscr{G}_{3}$. Then,

$$
\begin{aligned}
\gamma_{w}^{l} \int_{0}^{1} \Phi\left(\eta(t, \cdot, \alpha), c_{l}\right) d \alpha-\gamma_{w}^{r} \int_{0}^{1} \Phi\left(\eta(t, \cdot, \alpha), c_{r}\right) d \alpha \\
=\gamma_{w}^{l} \int_{0}^{1} \Phi\left(\eta(t, \cdot, \alpha), c_{l}\right) d \alpha-\gamma_{w}^{l} \int_{0}^{1} \Phi\left(\eta(t, \cdot, \alpha), c_{r}\right) d \alpha \\
\quad+\gamma_{w}^{l} \int_{0}^{1} \Phi\left(\eta(t, \cdot, \alpha), c_{r}\right) d \alpha-\gamma_{w}^{r} \int_{0}^{1} \Phi\left(\eta(t, \cdot, \alpha), c_{r}\right) d \alpha \\
=\gamma_{w}^{l} \int_{0}^{1}\left(\Phi\left(\eta(t, \cdot, \alpha), c_{l}\right)-\Phi\left(\eta(t, \cdot, \alpha), c_{r}\right)\right) d \alpha \\
\quad+\gamma_{w}^{l} \int_{0}^{1} \Phi\left(\eta(t, \cdot, \alpha), c_{r}\right) d \alpha-\gamma_{w}^{r} \int_{0}^{1} \Phi\left(\eta(t, \cdot, \alpha), c_{r}\right) d \alpha
\end{aligned}
$$


The first term in the right-hand side is nonnegative; indeed, the function $\tilde{u}(t, x):=$ $c_{l} \mathbb{1}_{\{x<0\}}+c_{r} \mathbb{1}_{\{x>0\}}$ is a classical Kruzhkov stationary solution of (10), whereas $k=\eta(t, x, \alpha)$ can be seen as the constant in the Kruzhkov definition. The last line in the above inequality is nonnegative thanks to (53), because $\left(c_{r}, c_{r}\right) \in$ $\mathscr{G}_{2}(F(t))$.

It remains to notice that inequalities (45) of Proposition 4.7imply $\left(D_{2}\right),\left(D_{3}\right)$ and the trace comparison inequalities (53) for $\left(c_{l}, c_{r}\right) \in \mathscr{G}_{1}(F(t)) \cup \mathscr{G}_{2}(F(t))$; this statement is contained in the proof of Proposition 3.1 in Appendix. Thus by Lemma 4.8 property $\left(D_{3}\right)$ also follows from the inequalities (45). Therefore, from Proposition 4.7 we derive that $u$ is a $\mathcal{G}$-entropy process solution of (1.3).

Now from Proposition 3.3 we readily obtain this final result:

Theorem 4.9. Under the CFL condition (38), the finite volume scheme 3436) converges in $\mathbf{L}_{\mathrm{loc}}^{p}(\Pi)$ for any $1 \leqslant p<+\infty$ to the unique $C G$-entropy solution of Problem (1]3) (which is also the unique $\mathcal{G}$-entropy solution of the problem).

\subsection{A strong compactness approach}

The tool of entropy process solutions is quite convenient for studying convergence of finite volume schemes, because it only requires an $\mathbf{L}^{\infty}$ estimate on the family of discrete solutions (for $\mathbf{L}^{\infty}$ weak- $\star$ compactness) and a "weak- $B V$ " estimate (for consistency). As such, it can be used for treating problems where strong compactness properties are lacking, e.g., multi-dimensional conservation laws on unstructured meshes (see [EGH00]).

Nonetheless, in many one-dimensional problems of the type (1) it is possible to obtain a uniform $B V$ estimate, and thus deduce the strong (pointwise, a.e.) compactness of approximate solutions, provided the data are of bounded variation. This is also the case of the constrained problem (13): in this context, we could apply the localized $B V$ estimate of Bürger and al. (see BGKT08, Lemma 4.2] and BKT09, Lemmas 5.3,5.4]) to deduce the strong compactness of the family of discrete solutions.

This makes it possible to study convergence of the finite volume methods for (13) with $B V$ data with the tools of [CG07, i.e. using the entropy inequalities (5) and the explicit constraint (6) (rewritten under the weak formulation (7)).

\section{$5 \quad$ Numerical results}

We now present some numerical experiments with the numerical scheme (34), using the Rusanov numerical flux Rus61:

$$
h(u, v)=\frac{f(u)+f(v)}{2}-\frac{\max \left(\left|f^{\prime}(u)\right|,\left|f^{\prime}(v)\right|\right)}{2}(v-u) .
$$

This numerical flux is consistent and monotone. The density of cars belongs to $[0,1]$ and the flux of the conservation law is $f(u)=u(1-u)$ in both cases, so that the constraint must belong to $[0,1 / 4]$. 


\subsection{A constrained Riemann problem}

This test case is very simple, it corresponds to the simulation of the solution to a Riemann problem. The domain of computation is $[-1 / 2,1 / 2]$ and the data are

$$
u_{0}(x)=\left\{\begin{array}{ll}
0.4 & \text { if } x<0, \\
0.5 & \text { if } x>0,
\end{array} \text { and } \quad F=0.2 .\right.
$$

The exact solution is composed of a classical shock wave with a negative speed, of a non-classical stationary shock wave at $x=0$ satisfying the constraint, and of another classical shock wave with a positive speed (see CG07 for more details on the solution of the Riemann problem). First, Figure \shows the comparison

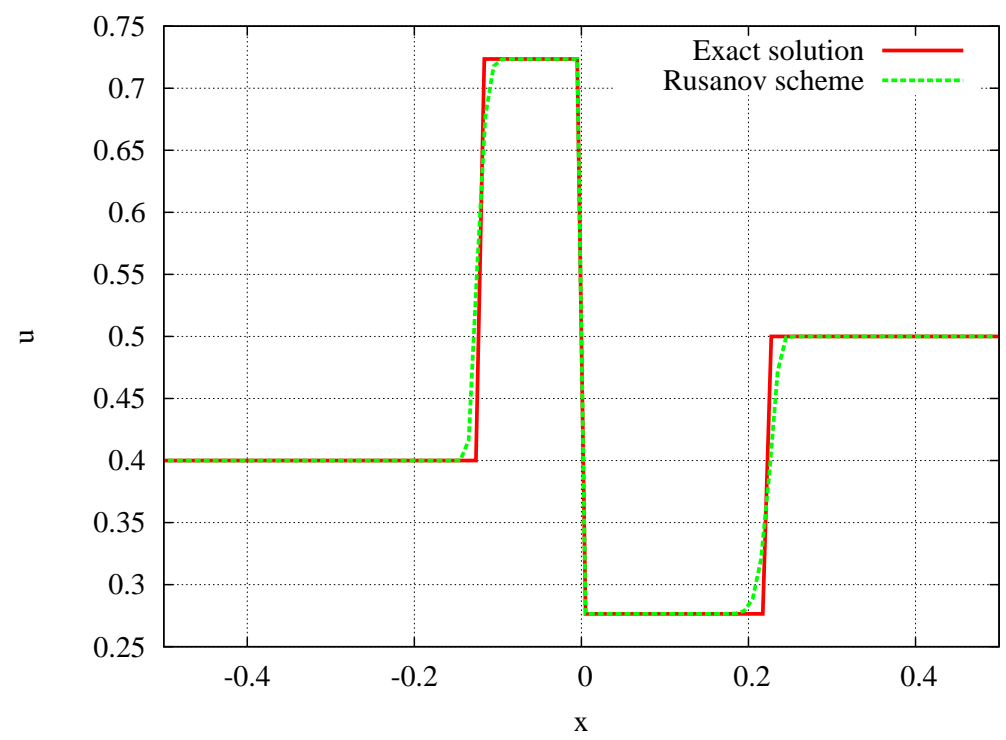

Figure 1: Comparison between the Rusanov scheme (100 cells, CFL=0.4) and the exact solution at time $t=1$.

between the numerical results provided by the Rusanov scheme and the exact solution. The non-classical shock wave seems to be perfectly solved. Still with this test case, an analysis of the convergence of the numerical scheme has been performed, as reported in the following table:

\begin{tabular}{|c|c|c|}
\hline Number of cells & $\mathbf{L}^{1}$-error & Rate of conv. \\
\hline 100 & $4.1938 \times 10^{-3}$ & - \\
300 & $1.2356 \times 10^{-3}$ & 1.112 \\
1000 & $3.7494 \times 10^{-4}$ & 0.990 \\
3000 & $1.1864 \times 10^{-4}$ & 1.047 \\
10000 & $3.6899 \times 10^{-5}$ & 0.970 \\
30000 & $1.2945 \times 10^{-5}$ & 0.953 \\
100000 & $3.6448 \times 10^{-6}$ & 1.053 \\
300000 & $1.2199 \times 10^{-6}$ & 0.996 \\
\hline
\end{tabular}


Figure2 depicts the error with respect to the space step. We can easily see that the rate of convergence is 1 , that is to say that the constraint does not affect the accuracy of the numerical scheme.

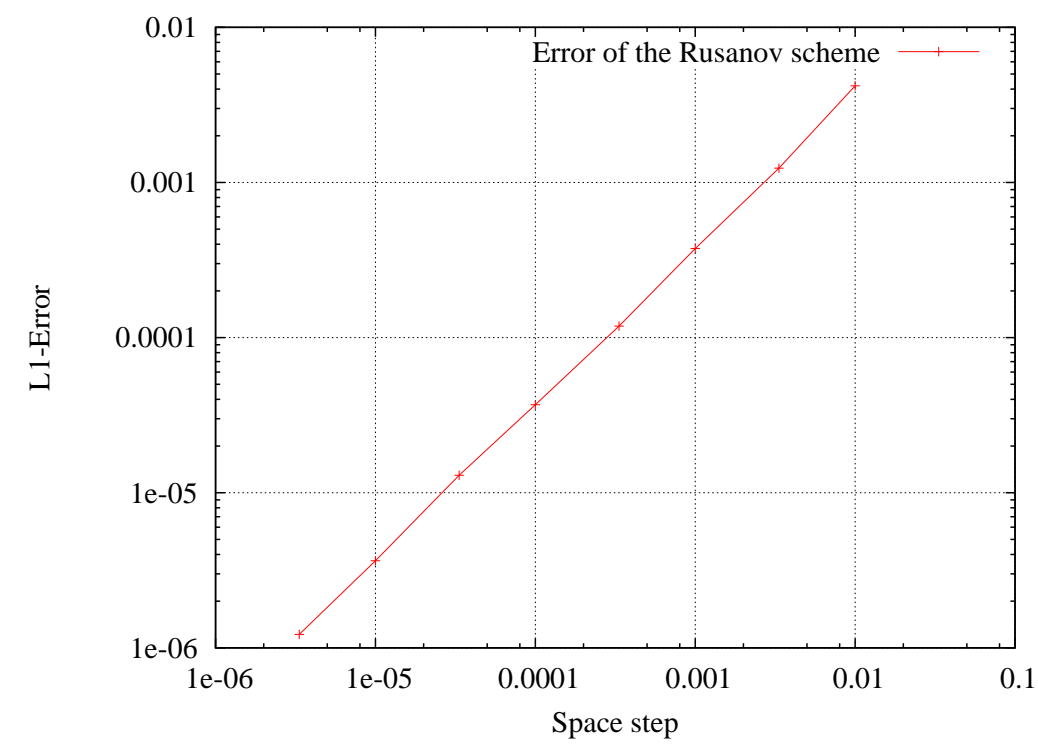

Figure 2: Convergence of the Rusanov scheme in the $\mathbf{L}^{1}$ norm.

\subsection{The green wave}

This test case is much more complicated. The space domain is $[0,100]$ and it involves five lights. They are modelled by the use of a constraint $F_{i}(t)$ for each light $i$. They are located at $x_{i}=(i+2) 12.5, i=1, \ldots, 5$. The constraint of the first light is defined by

$$
F_{1}(t)= \begin{cases}0 & \text { if } t \in[0,50) \\ \max _{u} f(u)=1 / 4 & \text { if } t \in[50,100)\end{cases}
$$

and is periodic (with period equal to 100). The other constraints are given by $F_{i}(t)=F_{1}(t-i \varphi), i=2, \ldots, 5$. The real constant $\varphi$ then corresponds to the time lag between the lights. Our aim is to minimize the influence of the lights $i$ with $i \geqslant 2$ on the traffic. The conditions of simulation are the following: the initial density is set to 0 on the whole domain, the left boundary condition is a Dirichlet condition, with $u(0, t)=0.1$, and at $x=100$, we impose an open boundary condition. The domain contains 1000 cells and the CFL number is set to 0.4 . Whatever the value of $\varphi$ is, the results become periodic in time, at least for $t \geqslant 500$. As an example, Figure 3 represents the evolution of the total mass in the domain for several values of $\varphi$. The case of "One light" corresponds to $F_{i} \equiv 1 / 4$ for $i \geqslant 2$ and is the ideal case.

In Figure 4 we can see the average over the time interval $[500,1000]$ of the total mass of cars and of the total variation (in space) of the velocity. 


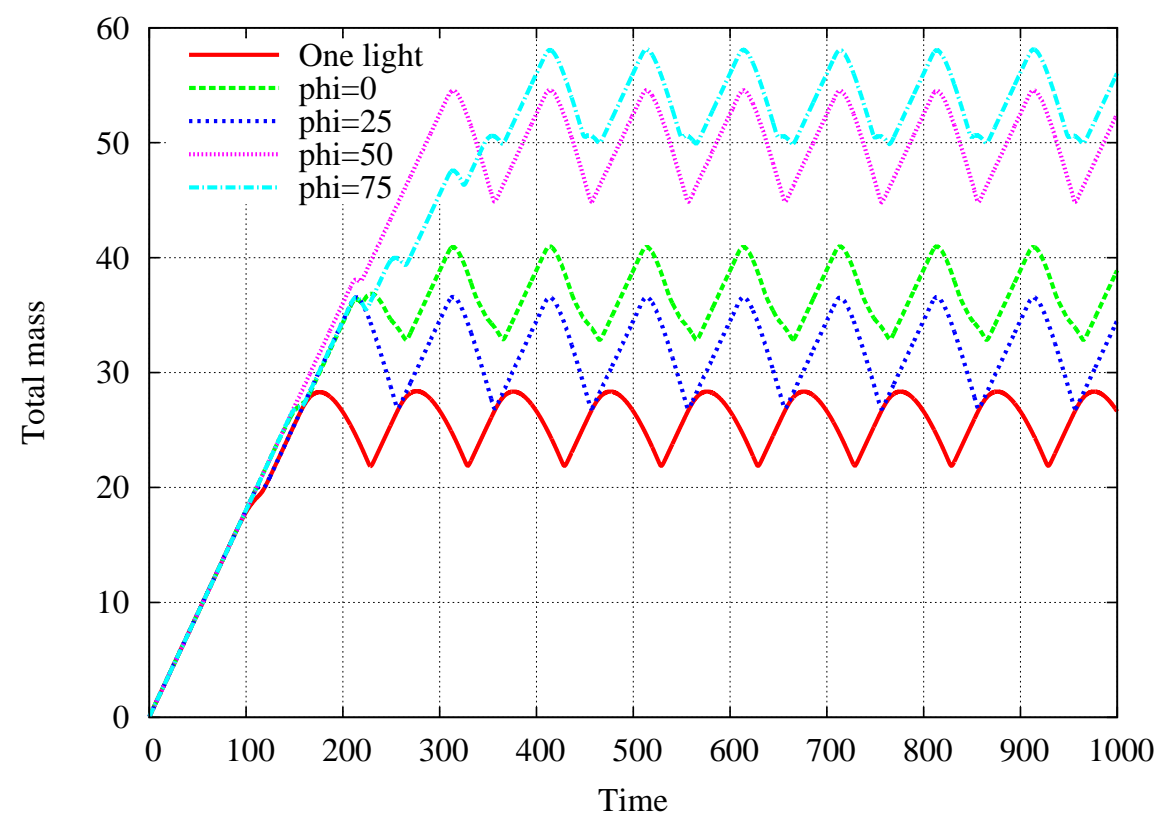

Figure 3: Time variation of the total mass, with different values of the time lag $\varphi$, compared to the ideal case (one light).

Average total mass

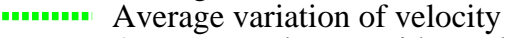

— Average total mass with one light

Average variation of velocity with one light

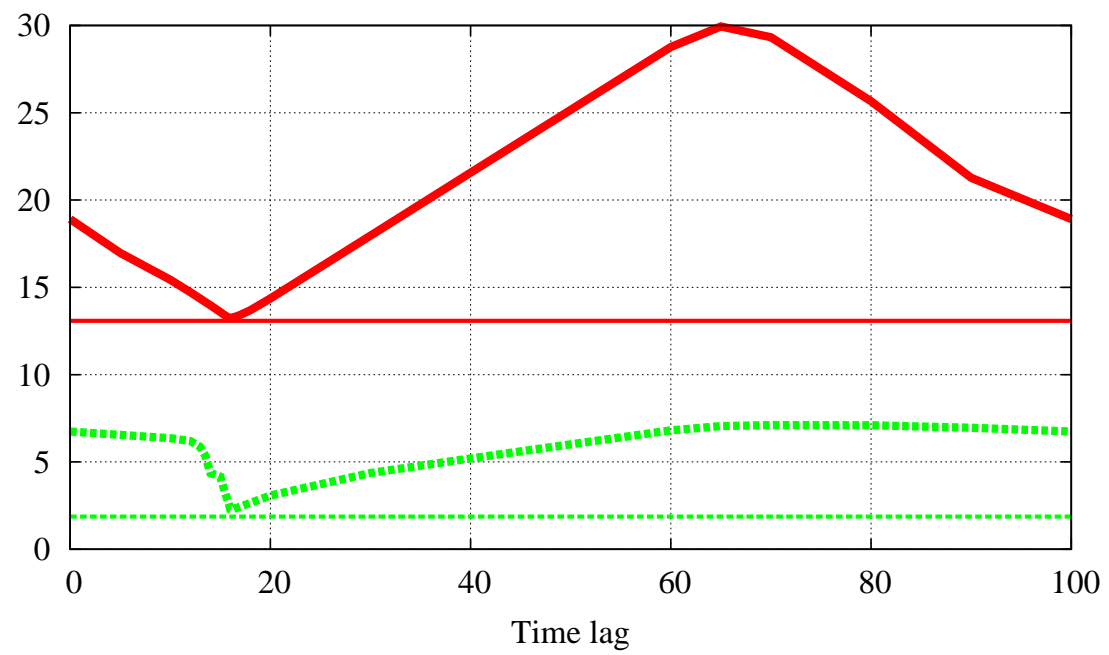

Figure 4: Variation of average quantities with respect to the time lag $\varphi$, compared to the ideal case (one light). 
One can see that $\varphi=16.1$ seems to be an optimal value. Moreover, for this value, the average total mass and the average total variation of the velocity are very close to the values obtained in the ideal case of one light (horizontal lines).

More results can be found in AGS, in particular the evolution of the density of cars, with different values of $\varphi$ (these figures have not been included here because their interpretation is not straightforward).

\section{Appendix: the postponed proofs}

Here we prove Lemma 2.7 Proposition 2.6 Proposition 2.10 and Proposition 3.1

Proof of Lemma 2.7

(i) We want to check that

$$
\forall\left(c_{l}, c_{r}\right),\left(b_{l}, b_{r}\right) \in \mathscr{G}(F) \quad \Phi\left(c_{l}, b_{l}\right) \geqslant \Phi\left(c_{r}, b_{r}\right) .
$$

- If $\left(c_{l}, c_{r}\right),\left(b_{l}, b_{r}\right) \in \mathscr{G}_{1}(F)$, then the two couples coincide; therefore we have $\Phi\left(c_{l}, b_{l}\right)=0=\Phi\left(c_{r}, b_{r}\right)$.

- If $\left(c^{l}, c^{r}\right) \in \mathscr{G}_{1}(F),\left(b^{l}, b^{r}\right) \in \mathscr{G}_{2}(F)$ and $b_{l}=b_{r} \leqslant B_{F}$, then

$$
\begin{aligned}
\Phi\left(c_{l}, b_{l}\right)-\Phi\left(c_{r}, b_{r}\right) & =\Phi\left(A_{F}, b_{l}\right)-\Phi\left(B(F), b_{l}\right), \\
& =f\left(A_{F}\right)-f\left(b_{l}\right)-f(B(F))+f\left(b_{l}\right)=0 .
\end{aligned}
$$

Similarly, if $\left(c_{l}, c_{r}\right) \in \mathscr{G}_{1}(F),\left(b_{l}, b_{r}\right) \in \mathscr{G}_{2}(F)$ and $b_{l}=b_{r} \geqslant A_{F}$, then

$$
\begin{aligned}
\Phi\left(c_{l}, b_{l}\right)-\Phi\left(c_{r}, b_{r}\right) & =\Phi\left(A_{F}, b_{l}\right)-\Phi\left(B_{F}, b_{l}\right), \\
& =f\left(b_{l}\right)-f(A(F))-f\left(b_{l}\right)+f\left(B_{F}\right)=0 .
\end{aligned}
$$

- If $\left(c_{l}, c_{r}\right) \in \mathscr{G}_{1}(F),\left(b_{l}, b_{r}\right) \in \mathscr{G}_{3}(F)$, then $b_{l} \leqslant B_{F}$ and $b_{r} \geqslant A_{F}$ and

$$
\begin{aligned}
\Phi\left(c_{l}, b_{l}\right)-\Phi\left(c_{r}, b_{r}\right) & =\Phi\left(A_{F}, b_{l}\right)-\Phi\left(B_{F}, b_{r}\right), \\
& =f\left(A_{F}\right)-f\left(b_{l}\right)+f\left(B_{F}\right)-f\left(b_{r}\right), \\
& =2\left(F-f\left(b_{l, r}\right)\right) \geqslant 0 .
\end{aligned}
$$

- If $\left(c_{l}, c_{r}\right),\left(b_{l}, b_{r}\right) \in \mathscr{G}_{2}(F) \cup \mathscr{G}_{3}(F)$, then the pairs $\left(b_{l}, b_{r}\right),\left(c_{l}, c_{r}\right)$ correspond to the Kruzhkov stationary solutions

$$
\tilde{b}(t, x):=b_{l} \mathbb{1}_{\{x<0\}}+b_{r} \mathbb{1}_{\{x>0\}}, \quad \tilde{c}(t, x):=c_{l} \mathbb{1}_{\{x<0\}}+c_{r} \mathbb{1}_{\{x>0\}}
$$

of the conservation law (10); inequality $\Phi\left(c_{l}, b_{l}\right)-\Phi\left(c_{r}, b_{r}\right) \geqslant 0$ is well known in this context (see Vol67).

The remaining cases are deduced by symmetry of $\Phi$; this proves (54).

(ii) Let us reason by contradiction. If $f\left(b_{l}\right)=f\left(b_{r}\right)$ but $\left(b_{l}, b_{r}\right) \notin \mathscr{G}(F)$, then either $f\left(b_{l, r}\right)>F$, or $f\left(b_{l, r}\right)<F$ with $b^{l}>b^{r}$. Pick $\left(c^{l}, c^{r}\right)=\left(A_{F}, B_{F}\right)$. In the first case we have $B_{F}<b_{l, r}<A_{F}$, whence

$$
\Phi\left(c_{l}, b_{l}\right)-\Phi\left(c_{r}, b_{r}\right)=f\left(A_{F}\right)-f\left(b_{l}\right)-f\left(B_{F}\right)+f\left(b_{r}\right)=2\left(F-f\left(b_{l, r}\right)\right)<0 .
$$


In the second case, $b_{r}<B_{F} \leqslant A_{F}<b_{l}$, and we get

$$
\Phi\left(c_{l}, b_{l}\right)-\Phi\left(c_{r}, b_{r}\right)=f\left(b_{l}\right)-f\left(A_{F}\right)+f\left(b_{r}\right)-f\left(B_{F}\right)=2\left(f\left(b_{l, r}\right)-F\right)<0 .
$$

Thus in both cases, we arrive to a contradiction with assumption (20).

Proof of Proposition [2.6. First, we claim the equivalence of (A) and (B).

Firstly, (A) implies $\left(B_{1}\right)$ and $\left(B_{3}\right)$, due to Lemma 2.7 (ii). Also $\left(B_{2}\right)$ follows from (A). Indeed, $\left(A_{2}\right)$ contains the Rankine-Hugoniot condition

$$
\left.\left.f\left(\gamma^{l} u\right)(t)\right)=f\left(\gamma^{r} u\right)(t)\right) \text { for a.e. } t>0 ;
$$

and $\left(A_{1}\right)$ contains the weak formulation of (11) in the domains $\{ \pm x>0\}$ (i suffices to take $\kappa=0$ and $\kappa=1$ in (15)). Splitting $\varphi=\varphi_{\varepsilon}^{1}+\varphi_{\varepsilon}^{2}$ (see the proof of Theorem 2.9) and letting $\varepsilon$ converge to zero, we derive $\left(B_{2}\right)$.

Secondly, $\left(B_{2}\right)$ implies the Rankine-Hugoniot condition (55); thus $\left(B_{3}\right)$ implies $\left(A_{2}\right)$, due to Lemma 2.7(ii). Since $\left(A_{1}\right)$ and $\left(B_{1}\right)$ coincide, this proves our first claim.

Now, we claim that $(\mathrm{A}) \Longrightarrow(\mathrm{C}) \Longrightarrow(\mathrm{B})$.

Firstly, starting from $\left(A_{1}\right)$ and using the expression of the one-sided traces $\gamma^{l, r}$ of $\Phi(u, \kappa), \kappa=c_{l, r}$, respectively, we derive the lower bound of the left-hand side of (19) of the form

$$
\mathcal{R}:=-\int_{0}^{+\infty}\left(\Phi\left(\left(\gamma^{l} u\right)(t), c_{l}\right)-\Phi\left(\left(\gamma^{r} u\right)(t), c_{r}\right)\right) \varphi(t, 0) d t .
$$

Further, $\mathcal{R}$ admits the right-hand side of (19) for lower bound. Indeed, for all $\left(b^{l}, b^{r}\right) \in \mathcal{G}(F(t))$,

$$
\Phi\left(\left(\gamma^{l} u\right)(t), b_{l}\right)-\Phi\left(\left(\gamma^{l} r u\right)(t), b_{r}\right) \geqslant 0
$$

due to $\left(A_{2}\right)$ and Lemma 2.7 i $)$; furthermore,

$$
\begin{array}{r}
\left|\left(\Phi\left(\left(\gamma^{l} u\right)(t), c_{l}\right)-\Phi\left(\left(\gamma^{r} u\right)(t), c_{r}\right)\right)-\left(\Phi\left(\left(\gamma^{l} u\right)(t), b_{l}\right)-\Phi\left(\left(\gamma^{l} r u\right)(t), b_{r}\right)\right)\right| \\
\leqslant M \operatorname{dist}\left(\left(c_{l}, c_{r}\right),\left(b_{l}, b_{r}\right)\right)
\end{array}
$$

for $M>0$ large enough, because $\Phi$ is Lipschitz continuous on $[0,1]^{2}$. Minimizing $\operatorname{dist}\left(\left(c_{l}, c_{r}\right),\left(b_{l}, b_{r}\right)\right)$ over all choices $\left(b_{l}, b_{r}\right) \in \mathcal{G}(F(t))$, we derive the global entropy inequality (19). Thus (C) follows from (A).

Now, starting from $(\mathbf{C})$ we readily get both $\left(B_{1}\right)$ and $\left(B_{2}\right)$. In order to prove $\left(B_{3}\right)$, we take the test function (13) in (19) and let $\varepsilon \rightarrow 0$. We derive

$$
\begin{aligned}
\int_{0}^{+\infty}\left(\Phi\left(\left(\gamma^{l} u\right)(t), c_{l}\right)-\Phi\left(\left(\gamma^{r} u\right)(t), c_{r}\right)\right) \varphi(t, 0) d t & \\
& \geqslant-M \int_{0}^{+\infty} \operatorname{dist}\left(\left(c_{l}, c_{r}\right), \mathcal{G}(F(t))\right) \varphi(t, 0) d t .
\end{aligned}
$$

Now take $\left(c_{l}, c_{r}\right) \in \mathcal{G}\left(F\left(t_{0}\right)\right)$, where $t_{0}$ is a Lebesgue point of each of the functions $F, \gamma^{l} u$ and $\gamma^{r} u$. Letting $\varphi$ approximate the Dirac function concentrated at $t=t_{0}$, at the limit we get the inequality $\Phi\left(\left(\gamma^{l} u\right)\left(t_{0}\right), c_{l}\right)-\Phi\left(\left(\gamma^{r} u\right)\left(t_{0}\right), c_{r}\right) \geqslant 0$. This justifies $\left(B_{3}\right)$. 
The proof of Proposition [2.6 is complete.

Proof of Proposition [2.10. We start from the entropy formulation (15). The method of doubling of variables of Kruzhkov, applied in the domains $\{ \pm x>0\}$, yields the so-called Kato inequality for the comparison of $u^{1}, u^{2}$ :

$$
\int_{\mathbb{R}^{+}} \int_{\mathbb{R}}\left(\left|u^{1}-u^{2}\right| \partial_{t}+\Phi\left(u^{1}, u^{2}\right) \partial_{x}\right) \varphi d x d t \geqslant 0
$$

for all $\varphi \in \mathcal{C}_{c}^{\infty}(\Pi \backslash\{x=0\}), \varphi \geqslant 0$.

Now, fix $R>0$ and replace $\varphi$ in this inequality by a sequence of approximations of the characteristic function of the set $\{t \in(0, T), 0<|x|<R+L(T-t)\}$, for instance $\varphi_{\varepsilon}(t, x)=\left(1-w_{\varepsilon}(x)\right) \chi_{\varepsilon}(t) \xi_{\varepsilon}(t, x)$ where

$$
\chi_{\varepsilon}(t)= \begin{cases}1 & \text { if } 0 \leqslant t<T, \\ \frac{T-t}{\varepsilon}+1 & \text { if } T \leqslant t<T+\varepsilon, \\ 0 & \text { if } t \geqslant T+\varepsilon,\end{cases}
$$

$w_{\varepsilon}$ is given by 13], and

$\xi_{\varepsilon}(t, x)= \begin{cases}1 & \text { if }|x| \leqslant R+L(T-t), \\ \frac{R+L(T-t)-|x|}{\varepsilon}+1 & \text { if } R+L(T-t) \leqslant|x|<R+L(T-t)+\varepsilon, \\ 0 & \text { if }|x| \geqslant R+L(T-t)+\varepsilon .\end{cases}$

This provides at the limit $\varepsilon \rightarrow 0$

$$
\begin{aligned}
& -\int_{-R}^{R}\left|u^{1}-u^{2}\right|(T, x) d x+\int_{-R-L T}^{R+L T}\left|u_{0}^{1}-u_{0}^{2}\right|(x) d x \\
& \quad+\int_{0}^{T}\left(\Phi\left(\left(\gamma^{r} u^{1}\right)(t),\left(\gamma^{r} u^{2}\right)(t)\right)-\Phi\left(\left(\gamma^{l} u^{1}\right)(t),\left(\gamma^{l} u^{2}\right)(t)\right)\right) d t \geqslant 0 .
\end{aligned}
$$

Fix $t_{0}>0$; without loss of generality, we can assume that $F^{1}\left(t_{0}\right) \geqslant F^{2}\left(t_{0}\right)$. Whenever this is convenient, we drop the dependency on $t_{0}$ in the subsequent notation. Recall that a CG-entropy solution also satisfies all the properties of (A),(B),(C) of Proposition 2.6 We make a case study quite similar to the one of the proof of Lemma 2.7

- If $\left(\gamma^{l} u^{i}, \gamma^{r} u^{i}\right) \in \mathscr{G}_{2}\left(F^{i}\right) \cup \mathscr{G}_{3}\left(F^{i}\right), i=1,2$, then both the standing waves

$$
\tilde{u}^{i}(t, x):=\left(\gamma^{l} u^{i}\right)\left(t_{0}\right) \mathbb{1}_{\{x<0\}}+\left(\gamma^{r} u^{i}\right)\left(t_{0}\right) \mathbb{1}_{\{x>0\}},
$$

$i=1,2$, are Kruzhkov entropy solutions of the (unconstrained) conservation law (11). Therefore we have the inequality

$$
\Phi\left(\gamma^{r} u^{1}, \gamma^{r} u^{2}\right)-\Phi\left(\gamma^{l} u^{1}, \gamma^{l} u^{2}\right) \leqslant 0
$$

which is well known since the work of Vol'pert Vol67.

- If $\left(\gamma^{l} u^{1}, \gamma^{r} u^{1}\right) \in \mathscr{G}_{1}\left(F^{1}\right)$ and $\left(\gamma^{l} u^{2}, \gamma^{r} u^{2}\right) \in \mathscr{G}_{2}\left(F^{2}\right) \cup \mathscr{G}_{3}\left(F^{2}\right)$, then we can use (18) to justify (57). Indeed, the definition of $\mathscr{G}_{j}$ and assumption $F^{1} \geqslant F^{2}$ lead to the inclusions $\mathscr{G}_{j}\left(F^{2}\right) \subset \mathscr{G}_{j}\left(F^{1}\right), j=2,3$. 
- If $\left(\gamma^{l} u^{1}, \gamma^{r} u^{1}\right) \in \mathscr{G}_{2}\left(F^{1}\right)$ and $\left(\gamma^{l} u^{2}, \gamma^{r} u^{2}\right) \in \mathscr{G}_{1}\left(F^{2}\right)$, then $\left(\gamma^{l} u^{2}, \gamma^{r} u^{2}\right)=$ $\left(A_{F^{2}}, B_{F^{2}}\right)$ and $\gamma^{l} u^{1}=\gamma^{r} u^{1}=: \gamma u^{1}$. We have to distinguish three cases:

- if $\gamma u^{1} \leqslant \gamma^{r} u^{2}$,

$$
\begin{aligned}
\Phi\left(\gamma^{r} u^{1}, \gamma^{r} u^{2}\right)- & \Phi\left(\gamma^{l} u^{1}, \gamma^{l} u^{2}\right) \\
& =\left(f\left(\gamma^{r} u^{2}\right)-f\left(\gamma u^{1}\right)\right)-\left(f\left(\gamma^{l} u^{2}\right)-f\left(\gamma u^{1}\right)\right)=0 ;
\end{aligned}
$$

- if $\gamma^{r} u^{2} \leqslant \gamma u^{1} \leqslant \gamma^{l} u^{2}$

$$
\begin{aligned}
& \Phi\left(\gamma^{r} u^{1}, \gamma^{r} u^{2}\right)-\Phi\left(\gamma^{l} u^{1}, \gamma^{l} u^{2}\right) \\
& \quad=\left(f\left(\gamma u^{1}\right)-f\left(\gamma^{r} u^{2}\right)\right)-\left(f\left(\gamma^{l} u^{2}\right)-f\left(\gamma u^{1}\right)\right) \leqslant 2\left(F^{1}-F^{2}\right) ;
\end{aligned}
$$

- if $\gamma u^{1} \geqslant \gamma^{l} u^{2}$

$$
\begin{aligned}
\Phi\left(\gamma^{r} u^{1}, \gamma^{r} u^{2}\right)- & \Phi\left(\gamma^{l} u^{1}, \gamma^{l} u^{2}\right) \\
= & \left(f\left(\gamma u^{1}\right)-f\left(\gamma^{r} u^{2}\right)\right)-\left(f\left(\gamma u^{1}\right)-f\left(\gamma^{l} u^{2}\right)\right)=0 .
\end{aligned}
$$

- If $\left(\gamma^{l} u^{1}, \gamma^{r} u^{1}\right) \in \mathscr{G}_{3}\left(F^{1}\right)$ and $\left(\gamma^{l} u^{2}, \gamma^{r} u^{2}\right) \in \mathscr{G}_{1}\left(F^{2}\right)$, we have $\gamma^{l} u^{1} \leqslant \bar{u} \leqslant$ $\gamma^{l} u^{2}, \gamma^{r} u^{2} \leqslant \bar{u} \leqslant \gamma^{r} u^{1}$. Assuming the strict inequalities $\gamma^{l} u^{1}<\gamma^{l} u^{2}$, $\gamma^{r} u^{2}<\gamma^{r} u^{1}$, we get

$$
\begin{aligned}
& \Phi\left(\gamma^{r} u^{1}, \gamma^{r} u^{2}\right)-\Phi\left(\gamma^{l} u^{1}, \gamma^{l} u^{2}\right) \\
& =\left(f\left(\gamma^{r} u^{1}\right)-f\left(\gamma^{r} u^{2}\right)\right)-\left(f\left(\gamma^{l} u^{2}\right)-f\left(\gamma^{l} u^{1}\right)\right) \leqslant 2\left(F^{1}-F^{2}\right) .
\end{aligned}
$$

Otherwise, at least one of the equalities $\gamma^{l} u^{1}=\bar{u}=\gamma^{l} u^{2}, \gamma^{r} u^{1}=\bar{u}=\gamma^{r} u^{2}$ holds; in this case, $F^{1}=F^{2}=F(\bar{u})$, and we get the conclusion (57).

- If $\left(\gamma^{l} u^{i}, \gamma^{r} u^{i}\right) \in \mathscr{G}_{1}\left(F^{i}\right), i=1,2$, then

$$
\Phi\left(\gamma^{r} u^{1}, \gamma^{r} u^{2}\right)-\Phi\left(\gamma^{l} u^{1}, \gamma^{l} u^{2}\right)=\Phi\left(A_{F^{1}}, A_{F^{2}}\right)-\Phi\left(B_{F^{1}}, B_{F^{2}}\right)=2\left(F^{1}-F^{2}\right) .
$$

Thus in all cases, we have

$$
\Phi\left(\left(\gamma^{r} u^{1}\right)\left(t_{0}\right),\left(\gamma^{r} u^{2}\right)\left(t_{0}\right)\right)-\Phi\left(\left(\gamma^{l} u^{1}\right)\left(t_{0}\right),\left(\gamma^{l} u^{2}\right)\left(t_{0}\right)\right) \leqslant 2\left|F^{1}-F^{2}\right|\left(t_{0}\right) .
$$

Hence

$$
\int_{-R}^{R}\left|u^{1}-u^{2}\right|(T, x) d x \leqslant \int_{0}^{T} 2\left|F^{1}-F^{2}\right|(t) d t+\int_{-R-L T}^{R+L T}\left|u_{0}^{1}-u_{0}^{2}\right|(x) d x
$$

letting $R$ tend to $+\infty$, we conclude the proof.

Proof of Proposition 3.1. The proof is similar to the one of Proposition 2.6.

In order to show that (D) implies $(\mathbf{E})$, we first take $\varphi(t, x)\left(1-w_{\varepsilon}(x)\right)$ for the test function in (27), where $\varphi \in \mathcal{C}_{c}^{\infty}(\Pi)$ and $w_{\varepsilon}$ is given by (13). Letting $\varepsilon$ decrease to zero and using the definition of weak traces in $\left(D_{3}\right)$, we get the inequality

$$
\begin{aligned}
& I \geqslant \int_{0}^{+\infty} D\left(t,\left(c_{l}, c_{r}\right)\right) \varphi(t, 0) d t \\
& D\left(t,\left(c_{l}, c_{r}\right)\right):=\gamma_{w}^{l}\left[\int_{0}^{1} \Phi\left(\mu(\cdot, \alpha), c_{l}\right) d \alpha\right](t)-\gamma_{w}^{r}\left[\int_{0}^{1} \Phi\left(\mu(\cdot, \alpha), c_{r}\right) d \alpha\right](t),
\end{aligned}
$$


where $I$ denotes the left-hand side of the global entropy inequality (30). Fix $t>0$ and let $\left(b_{l}, b_{r}\right) \in \mathcal{G}(F(t))$. Then we can write $D\left(t,\left(c_{l}, c_{r}\right)\right)$ as the sum of three terms:

$$
\begin{aligned}
D\left(t,\left(c_{l}, c_{r}\right)\right)= & \left(\gamma_{w}^{l} \int_{0}^{1} \Phi\left(\mu(t, \cdot, \alpha), b_{l}\right) d \alpha-\gamma_{w}^{r} \int_{0}^{1} \Phi\left(\mu(t, \cdot, \alpha), b_{r}\right) d \alpha\right) \\
+ & \left.\gamma_{w}^{l}\left(\int_{0}^{1} \Phi\left(\mu(t, \cdot, \alpha), c_{l}\right) d \alpha-\int_{0}^{1} \Phi\left(\mu(t, \cdot, \alpha), b_{l}\right)\right) d \alpha\right) \\
& \left.-\gamma_{w}^{r}\left(\int_{0}^{1} \Phi\left(\mu(t, \cdot, \alpha), c_{r}\right) d \alpha-\int_{0}^{1} \Phi\left(\mu(t, \cdot, \alpha), b_{r}\right)\right) d \alpha\right) .
\end{aligned}
$$

By property $\left(D_{3}\right)$, the first term in the right-hand side of (59) is non-negative. The set $\mathcal{G}(F(t))$ being compact, we can pick the couple $\left(b_{l}, b_{r}\right)$ which realizes the distance $\operatorname{dist}\left(\left(c_{l}, c_{r}\right), \mathcal{G}(F(t))\right)$. The function $\Phi$ is Lipschitz continuous; this permits to bound the two other terms in the right-hand side of (59) by $M \operatorname{dist}\left(\left(c_{l}, c_{r}\right), \mathcal{G}(F(t))\right)$, for some $M>0$. Hence (30) follows.

Conversely, (E) implies (D). The assertions $\left(D_{1}\right)$ and $\left(D_{2}\right)$ are straightforward. For the proof of $\left(D_{3}\right)$, we pick $\xi(t) w_{\varepsilon}(x)$ in (13) for the test function in the global entropy inequality [30], $\xi \in \mathcal{C}_{c}^{\infty}((0,+\infty)), \xi \geqslant 0$, and let $\varepsilon$ tend to 0 . As a result, we get

$$
\int_{0}^{+\infty} D\left(t,\left(c_{l}, c_{r}\right)\right) \xi(t) d t \geqslant-M \int_{0}^{+\infty} \operatorname{dist}\left(\left(c_{l}, c_{r}\right), \mathcal{G}(F(t))\right) \xi(t) d t
$$

where $D\left(t,\left(c_{l}, c_{r}\right)\right)$ is defined in (58). Take a common Lebesgue point $t_{0}$ of $F$ (it is also a Lebesgue point of the map $t \mapsto \operatorname{dist}\left(\left(c_{l}, c_{r}\right), \mathcal{G}(F(t))\right)$, for all $\left.\left(c_{l}, c_{r}\right) \in[0,1]^{2}\right)$ and of the map $t \mapsto\left\{\left(c_{l}, c_{r}\right) \mapsto D\left(t,\left(c_{l}, c_{r}\right)\right)\right\} \in C\left([0,1]^{2}\right)$ (see Remark [6). Letting $\xi$ converge to the Dirac mass concentrated at the point $t_{0}$, we get

$$
\forall\left(c_{l}, c_{r}\right) \in[0,1]^{2} \quad D\left(t_{0},\left(c_{l}, c_{r}\right)\right) \geqslant \operatorname{dist}\left(\left(c_{l}, c_{r}\right), \mathcal{G}\left(F\left(t_{0}\right)\right)\right) .
$$

Picking $\left(c_{l}, c_{r}\right) \in \mathcal{G}\left(F\left(t_{0}\right)\right)$, we deduce the claim of $\left(D_{3}\right)$.

Acknowledgement This paper was written as part of the international research program on Nonlinear Partial Differential Equations at the Centre for Advanced Study at the Norwegian Academy of Science and Letters in Oslo during the academic year 2008-09. The first and third authors are grateful to Kenneth H. Karlsen for the invitation to Oslo and for stimulating discussions. The second author was partially supported by INRIA Sophia Antipolis Méditerranée.

\section{References}

[AMG05] Adimurthi, S. Mishra, and G.D. Veerappa Gowda. Optimal entropy solutions for conservation laws with discontinuous flux-functions. $J$. Hyperbolic Diff. Eq., 2(4):783-837, 2005. 
[AKR $\quad$ B. Andreianov, K.H. Karlsen, and N.H. Risebro. A theory of $\mathbf{L}^{1}$-dissipative solvers for scalar conservation laws with discontinuous flux. In preparation.

[AGS] B. Andreianov, P. Goatin, and N. Seguin. Simulations of the green wave. http://www.ann.jussieu.fr/publications/2009.php3 and http://www.ann.jussieu.fr/ $\sim_{\text {seguin/TMP/green-wave.html }}$

[AuP05] E. Audusse and B. Perthame. Uniqueness for scalar conservation laws with discontinuous flux via adapted entropies. Proc. Roy. Soc. Edinburgh Sect. A 135(2):253-265, 2005.

[BV06] F. Bachmann and J. Vovelle. Existence and uniqueness of entropy solution of scalar conservation laws with a flux function involving discontinuous coefficients. Comm. Partial Differential Equations, 31:371$395,2006$.

[BJ97] P. Baiti and H.K. Jenssen. Well-posedness for a class of $2 \times 2$ conservation laws with $L^{\infty}$ data. J. Diff. Eq. 140(1):161-185, 1997.

[BGKT08] R. Bürger, A. García, K.H. Karlsen and J.D. Towers. A family of numerical schemes for kinematic flows with discontinuous flux. $J$. Engrg. Math. 60 (2008), no. 3-4, 387-425.

[BKT09] R. Bürger, K. H. Karlsen, and J. D. Towers. An Engquist-Osher-type scheme for conservation laws with discontinuous flux adapted to flux connections. SIAM J. Num. Analysis, to appear.

[CF99] G.-Q. Chen and H. Frid. Divergence-measure fields and hyperbolic conservation laws. Arch. Ration. Mech. Anal., 147(2):89-118, 1999.

[CG07] R. M. Colombo and P. Goatin. A well posed conservation law with a variable unilateral constraint. J. Differential Equations, 234(2):654$675,2007$.

[DiP85] R. J. DiPerna. Measure-valued solutions to conservation laws. Arch. Rational Mech. Anal., 88(3):223-270, 1985.

[EGH00] R. Eymard, T. Gallouët, and R. Herbin. Finite volume methods. In Handbook of numerical analysis, Vol. VII, Handb. Numer. Anal., VII, pages 713-1020. North-Holland, Amsterdam, 2000.

[KRT03] K.H. Karlsen, N.H. Risebro and J.D. Towers. $\mathbf{L}^{1}$ stability for entropy solutions of nonlinear degenerate parabolic convection-diffusion equations with discontinuous coefficients. Skr. K. Nor. Vid. Selsk. (2003), $49 \mathrm{pp}$.

[Kru70] S.N. Kružkov. First order quasilinear equations with several independent variables. (Russian), Mat. Sb., 81(2):, 228-255, 1970. English transl. in Math. USSR Sb., 10 (1970).

[Pan93] E. Yu. Panov. Strong measure-valued solutions of the Cauchy problem for a first-order quasilinear equation with a bounded measure-valued initial function. Moscow Univ. Math. Bull. 48(1):18-21, 1993. 
[Pan07] E. Yu. Panov. Existence of strong traces for quasi-solutions of multidimensional conservation laws. J. Hyperbolic Differ. Equ., 4(4):729-770, 2007.

[Rus61] V. V. Rusanov. The calculation of the interaction of non-stationary shock waves with barriers. Ž. Vyčisl. Mat. i Mat. Fiz., 1:267-279, 1961.

[SV03] N. Seguin and J. Vovelle. Analysis and approximation of a scalar conservation law with a flux function with discontinuous coefficients. Math. Models Methods Appl. Sci., 13(2):221-257, 2003.

[Sze89] A. Szepessy. Convergence of a shock-capturing streamline diffusion finite element method for a scalar conservation law in two space dimensions. Math. Comp., 53(188):527-545, 1989.

[Tow00] J. D. Towers. Convergence of a difference scheme for conservation laws with a discontinuous flux. SIAM J. Numer. Anal., 38(2):681-698 (electronic), 2000.

[Vas01] A. Vasseur. Strong traces of multidimensional scalar conservation laws. Arch. Ration. Mech. Anal., 160(3):181-193, 2001.

[Vol67] A. I. Vol'pert. Spaces $B V$ and quasilinear equations. Mat. Sb. (N.S.), 73(115):255-302, 1967.

Boris ANDREIANOv boris.andreianov@univ-fcomte.fr

Laboratoire de Mathématiques

Université de Franche-Comté

16 route de Gray

25030 Besançon Cedex

France

Paola GoAtin goatin@univ-tln.fr

ISITV

Université du Sud Toulon - Var

Avenue Georges Pompidou

BP 56

83162 La Valette du Var Cedex

France

Nicolas SEGUIN seguin@ann.jussieu.fr

Laboratoire J.-L. Lions

UPMC Univ Paris 06

BC 187

75252 Paris cedex 05

France 